\title{
Accurate Prediction of the Wall Shear Stress in Rod Bundles with the Spectral Element Method at High Reynolds Numbers
}

\author{
Justin Walker, Elia Merzari, Aleks Obabko, Paul Fischer, and Andrew Siegel \\ E-mail: emerzari@anl.gov \\ Mathematics and Computer Science Division, Argonne National Laboratory
}

August 8, 2014

\begin{abstract}
Resolving flow near walls is critical to reproducing the high rates of shear that generate turbulence in high Reynolds number, wall-bounded flows. In the present study, we examine the resolution requirements for correctly reproducing mean flow quantities and wall shear stress distribution in a large eddy simulation using the spectral element method. In this method, derivatives are only guaranteed in a weak sense, and the same is true of quantities composed of derivatives, such as the wall shear stress. We are interested in what is required to resolve the wall shear stress in problems that lack homogeneity in at least one direction. The problem of interest is that of parallel flow through a rod bundle configuration. Several meshes for this problem are systematically compared. In addition, we conduct a study of channel flow in order to examine the issues in a canonical flow that contains spanwise homogeneity missing in rod bundle flow. In the case of channel flow, we compare several meshes and subgrid scale models. We find that typical measures of accuracy, such as the law of the wall, are not sufficient for determining the resolution of quantities that vary along the wall. Spanwise variation of wall shear stress in underresolved flows is characterized by spikes - physical points without well-defined derivatives of the velocity-found at element boundaries. These spikes are not particular to any subgrid scale model and are the unavoidable consequence of underresolution. Accurately reproducing the wall shear stress distribution, while minimizing the computational costs, requires increasing the number of elements along the wall (local $h$-refinement) and using very high order $(N=19)$ basis functions ( $p$-refinement). We suggest that while these requirements are not easily generalized to grid spacing guidelines, one can apply a general process: construct a mesh that progessively increases elements along any walls, and increase the order of basis functions until the distribution of wall shear stress or any other quantity of interest is smooth.
\end{abstract}




\section{Introduction}

Accurate prediction of wall shear stress is critical in the analysis of turbulent wall-bounded flows [24]. It is important for the production of correct friction factors, turbulent kinetic energy production, the analysis of heat transfer, and study of fluid-structure interaction. In most flows of engineering interest, the wall shear stress and viscous stresses are nonuniform since they are functions of the velocity derivatives. In order to predict the wall shear stress correctly, the regularity and smoothness of the velocity solution need to be guaranteed. However, in methods that solve the weak form of the Navier-Stokes equation such as the spectral element method (SEM), the space of solutions is usually chosen to be less regular than $C^{1}$ so that

only weak derivatives are guaranteed to exist, and the derivatives do not have the same strict requirement to converge that the solution does [5]. While approximations of the convergence of derivatives exist, we are interested in what is seen in practice, especially in the case of underresolved, non-DNS (direct numerical simulation) flows. We aim to provide guidelines to predict wall shear stress in complex engineering flows at realistic Reynolds numbers while maintaining the mesh resolution as coarse as possible. The target problem chosen is the parallel flow in rod bundles.

The prediction of the flow in rod bundles is of fundamental importance in a variety of engineering fields. It is, for instance, relevant for heat transfer applications such as the design of tube and shell heat exchangers as well as nuclear reactor core analysis. In nuclear reactor cores, the heat produced by the nuclear fuel contained within the rods is removed by the coolant flowing through the bundle. To predict with greater accuracy the behavior and the thermal performance of nuclear reactors, one must predict accurately the underlying hydrodynamics.

Rod bundle flows differ significantly from pipe flow and parallel channel flow 13,26 32]. Rod bundles present flow characteristics that are reminiscent of external flows. Viscous dissipation and wall shear stress change azimuthally. Coherent structures might develop in the streamwise direction for particularly tight bundles. Moreover, the flow anisotropy induces secondary flows.

Especially important for nuclear engineering applications is the prediction of the peak fuel temperature and peak fluid temperature. Ideally, one would compute a temperature solution by prescribing a heat flux at the boundary or, even better, by performing a conjugate heat transfer calculation. In cases where that is not possible, the Reynolds analogy can be used to obtain an approximation to the heat transfer from known values of the wall shear stress.

RANS models developed for internal flows do not usually fare well for rod bundles because of a combination of the previous points [1, 19]. Accounting for more physics and reasonable tuning leads to better 
results. However, a more general approach such as large eddy simulation (LES) is desirable to lead to better predictability and freedom in exploring the design space [18]. In LES the large scales of turbulence are resolved while the small scale contribution to dissipation is modeled. Wall-resolved LES presents significant advantages when considering conjugate heat transfer calculations. Moreover, it permits exploring the physics of near-wall turbulence, which dramatically influences heat transfer. Traditionally, wall-resolved LES has been limited to relatively low Reynolds numbers since the computational cost scales superlinearly with the Reynolds number. One of the purposes of the present work is to test such methodology at Reynolds numbers of engineering interest and to verify how coarse the grid can be. As part of the Center for the Exascale Simulation of Advanced Reactors (CESAR) [31] effort, increasingly large simulations are planned comprising full reactor cores, and accessing the minimal computational requirements is of fundamental importance.

The code used for all the calculations performed for the present work is Nek5000 7$]$, a code developed at Argonne National Laboratory and the target CFD code of CESAR. Nek5000 has demonstrated excellent parallel performance on petascale level machines when at least 20,000 collocation points are allocated per MPI process. Nek5000 solves the incompressible Navier-Stokes equations in the weak form using the spectral element method, a higher-order method in space.

In the present paper we explore the computational requirements to perform LES simulations at high Reynolds numbers in rod bundles using Nek5000. We study two cases: parallel flow around a single rod in an infinite array and channel flow. The objective is to be able to perform full bundle simulations at the Reynolds numbers encountered in prototype reactors $(\sim 70,000)$ with the least amount of computational effort possible while maintaining excellent accuracy for both velocity distribution and wall shear stress. This implies operating LES at the limit of underresolution, where the method does not guarantee a smooth solution of the derivatives. An approach to locally increase the resolution was found to be required in order to correctly resolve the wall shear stress. In fact, in order to achieve accurate prediction of wall shear stress in an LES, resolution requirements are greater than what is needed for other measures of accuracy such as the law of the wall.

Preliminary results for a 37-rod bundle simulation, performed by using the resolution guidelines described in the present work, are shown in Figure 1. The grid resolution studies presented in this work have been performed on an infinite array (Figure 1d) for simplicity and reduced computational cost.

In Section 2, the computational methodology is summarized. In Section 3 the issues concerning wall shear stress are described in more detail. Section 4 contains a systematic study of the effects of underresolution on the mean flow quantities in the case of channel flow. Section 5 focuses on the target case, the parallel flow in 
rod bundles. The results of several calculations are compared and conclusions drawn about best practices.
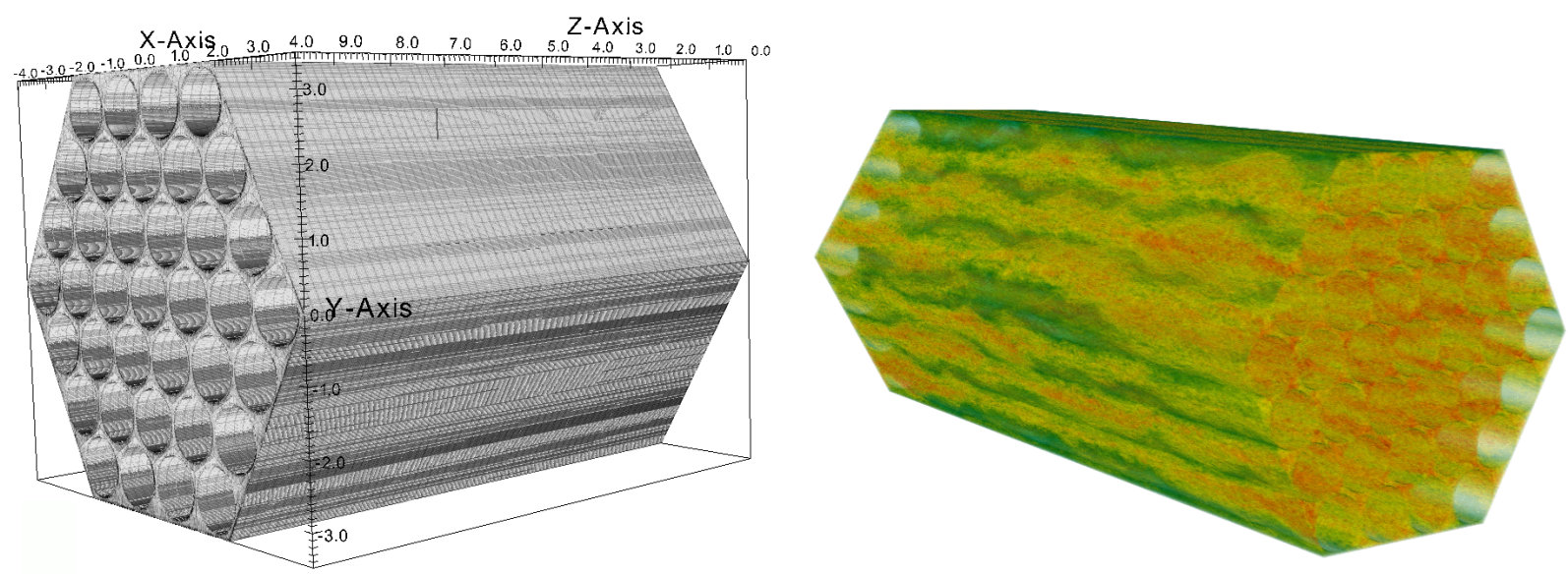

(a) Mesh for the 37-rod bundle case

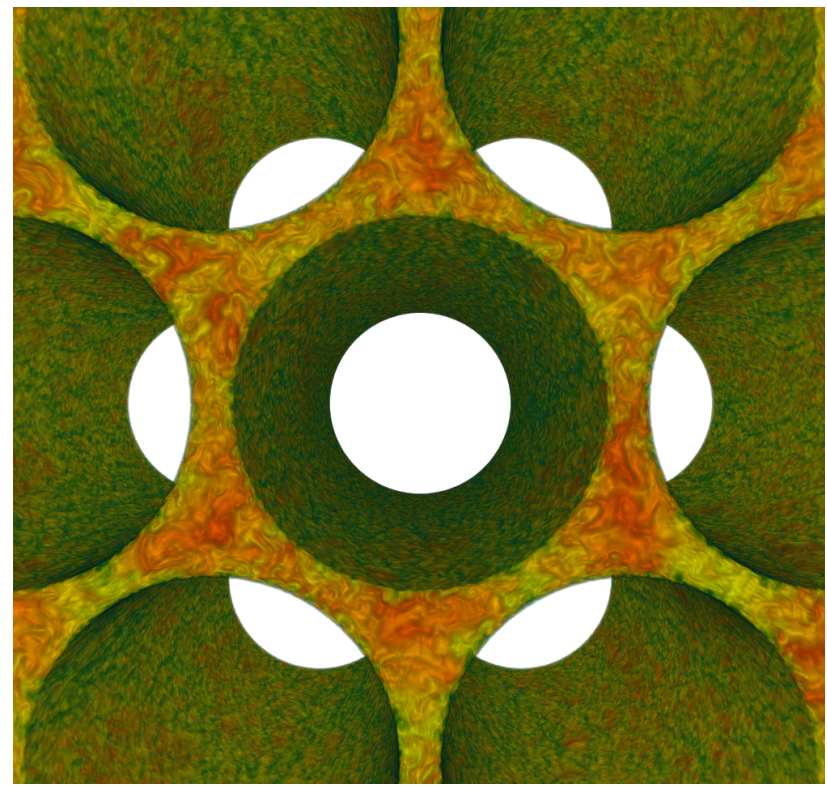

(b) Volume rendering of the streamwise velocity

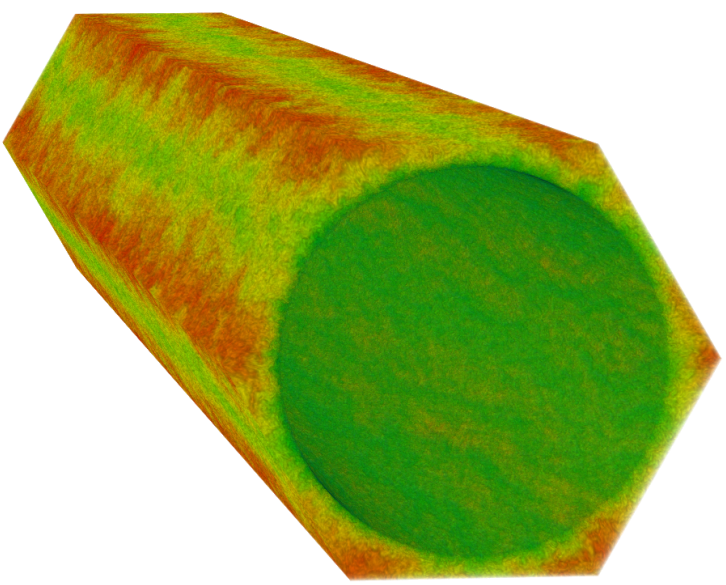

(c) Volume rendering of the streamwise velocity, detail

(d) Volume rendering of the single rod arrangement

Figure 1: Preliminary results of a 37-rod bundle case in the first three figures, along with results of the single pin analyzed in the present study in the last figure.

\section{Methodology}

Calculations were performed by using the spectral element code Nek5000 [9]. Nek5000 solves the incompressible Navier-Stokes equations. 
In this study the incompressible Navier-Stokes equations are solved in their standard Cartesian form:

$$
\begin{aligned}
\frac{\partial u_{i}}{\partial t}+u_{j} \frac{\partial u_{i}}{\partial x_{j}} & =-\frac{\partial p}{\partial x_{i}}+\frac{1}{\operatorname{Re}} \frac{\partial^{2} u_{i}}{\partial x_{j} \partial x_{j}} \\
\frac{\partial u_{i}}{\partial x_{i}} & =0
\end{aligned}
$$

along with appropriate boundary conditions. All variables have been nondimensionalized by a characteristic length $\delta$ and bulk velocity scale $U_{b}$; Re is the Reynolds number.

The domain and equations are discretized following the form of the spectral element method. The spectral element method solves the Navier-Stokes equations on a given domain by discretizing the solution space into elements such that the global solution is composed of piecewise polynomial functions with compact support. The problem is solved as a variational problem in what is known as weak form [5]. (More details are given in Section 3.) Lagrangian polynomial functions of up to the 23rd degree have been used to discretize the velocity field in each element in the present work. In the generalized, weighted residual framework, the present spectral-element method can be classified as a Galerkin method where the test functions and the basis functions for each element are Lagrange polynomials evaluated on Gauss-Lobatto-Legendre collocations points for the velocity. The pressure is solved with the same order polynomials as the velocity $\left(P_{N}-P_{N}\right.$ formulation).

Equations (1a) and (1b) are integrated in time by using a characteristic scheme as described by Maday et al. [17]. This method avoids the Courant-Friedrichs-Lewy (CFL) stability constraint in typical semi-implicit timestepping implementations, allowing greater time steps with CFL values in the range of 3-4.

In all problems considered here, periodic boundary conditions are applied in the streamwise direction. A dynamic forcing term is calculated at each time step to ensure a fixed flow rate.

The LES calculations carried out in this study use the stabilizing filter of Fischer and Mullen [8]. In this method, the solution at each time step is explicitly filtered. The filter operator $F_{\alpha}$ is defined as

$$
F_{\alpha}:=\alpha I_{N-1}+(1-\alpha) \mathbf{I}
$$

where $\mathbf{I}$ is the identity operator and $I_{N}$ is the interpolation operator at the $N+1$ GLL nodes. This filter has the desirable property that it preserves the spectral convergence of SEM. As $N \rightarrow \infty$, the interpolation error goes to zero exponentially.

Note that in Sections 4 and 5 of this paper we decompose the $x$ component of velocity as $\tilde{u}=U+u$, 
where $U$ is the mean component and $u$ is the fluctuating component, and use $\bar{u}$ to indicate averaging of a fluctuating component. Similarly, the $y$ and $z$ components of velocity are denoted by $\tilde{v}=V+v$ and $\tilde{w}=W+w$, respectively.

\section{Wall Shear Stress}

Nek5000 solves the Navier-Stokes equations using the weak formulation. Given a boundary value problem on a domain $\Omega$ with a solution $u \in V$,

$$
D[u(x)]=f, \quad x \in \Omega
$$

where $D$ is a differential operator, one can recast the problem in a variational form [5], also know as the weak formulation. In this form, one wants to find $u \in V$ such that

$$
(D u, v)=(f, v), \quad \forall v \in V
$$

where $(\cdot, \cdot)$ is a suitable inner product and $v$ is a test function. One generally chooses the solution space $V \subset H_{0}^{1}(\Omega)^{d}$, which is the Sobolev space of functions in $L^{2}$ that vanish on the boundary and whose first derivatives are in $L^{2}$, where $d$ is the dimension.

In applications, one generally uses analytic functions of either polynomials or trignometric functions to approximate the unique solution $\hat{u}$. Let $\left\{V_{N}\right\}_{N=0}^{\infty}$ represent a family of finite-dimensional subspaces that cover $V$. We then look for approximate solutions $u_{N} \in V_{N}$. With Galerkin methods, such as SEM, the solution space is $V_{N}:=\mathbb{P}_{N}(\Omega)$, the space of polynomials of order $N$.

The choice of $V$ has implications for the error of the approximate solution and, more important, its derivative, as found by the weak form of the problem. Sobolev embedding theorems [25] tell us that the Sobolev space given by

$$
W^{1, p}(\Omega):=\left\{v \in L^{p}(\Omega): \frac{\partial v}{\partial x_{i}} \in L^{p}(\Omega), i=1, \ldots, d\right\}
$$

is embedded $W^{1, p}(\Omega) \subset C(\Omega)$ as long as $p>d$. This case obviously holds for $d=1$, implying that all solutions are continuous. Less regularity holds for $d \geq 2$, since the existence of finite $L^{p}$ norms for $p>2$ cannot be guaranteed. 
The approximation error from using a truncated polynomial expansion for $d=1$, as in SEM, is given as follows. For any element $u \in H_{w}^{1}(\Omega)$, the truncated Legendre polynomial expansion $P_{N} u:=\sum_{k=0}^{N} \hat{u}_{k} p_{k}$ converges [5] as

$$
\left\|u-P_{N} u\right\|_{L_{w}^{2}(\Omega)} \leq C N^{-m}\|u\|_{H_{w}^{m} \Omega} .
$$

More important for the current discussion is the approximation error for the derivatives $(\cdot)_{x}:=\partial(\cdot) / \partial x$ given by

$$
\left\|u_{x}-\left(P_{N} u\right)_{x}\right\|_{L_{w}^{2}(\Omega)} \approx C N^{3 / 2-m}
$$

which means that if $u \in H_{w}^{1}(\Omega)$, but $u \notin H_{w}^{2}(\Omega)$ then $\left(P_{N} u\right)_{x}$ does not necessarily converge to $u_{x}$. The scenario is even worse for $d \geq 2$.

The wall shear stress is defined by

$$
\tau_{w}:=\left[\left(\tau_{i j} n_{j}\right)^{2}-\left(\tau_{i j} n_{i} n_{j}\right)^{2}\right]^{\frac{1}{2}}
$$

where $n$ is the unit normal of a boundary surface and $\tau_{i j}$ is the stress tensor, defined as

$$
\tau_{i j}=\mu\left(\frac{\partial u_{i}}{\partial x_{j}}+\frac{\partial u_{j}}{\partial x_{i}}\right)
$$

where $\mu$ is the dynamic viscosity. When there is spanwise homogeneity, such as in the case of turbulent channel flow, the definition of the wall shear stress simplifies to another standard form,

$$
\tau_{w}=\mu \frac{\partial u}{\partial n}
$$

where $n$ is the normal direction to a boundary.

The results from this section highlight the difficulty of correctly reproducing the wall shear stress. Since the wall shear stress is derived from derivatives of the solution, it is important that these be correct. In particular, in a weak formulation, where regularity of the derivatives is not guaranteed, the resolution requirements to resolve the derivatives of the solution may be more stringent than those to resolve the solution to a given level of accuracy. 


\section{Channel flow}

The effects of underresolution on the wall shear stress were investigated in the canonical case of turbulent channel flow. We compare the results of high and low amounts of filtering and of higher-order and lowerorder spectral elements. Channel flow has been studied extensively at low Reynolds numbers. For example, two calculations are the DNS by Kim, Moin, and Moser [15] with Re $=3300$ and the DNS of Moser, Kim, and Mansour [22] with Re $=10935$, hereafter KMM and MKM, respectively. We will compare our results with the higher Reynolds number study of MKM.

The focus of these comparisons is the local wall shear stress as a function of the spanwise position, which is the relevant quantity of interest for rod bundle flows.

\subsection{Numerical Model}

The domain of the problem is a rectangular channel periodic in the streamwise and spanwise directions, $x$ and $z$, respectively. The last two boundaries, normal to the $y$ axis at $y= \pm \delta$, have the no-slip boundary condition applied. All quantities have been nondimensionalized by the channel half-width $\delta$ and mean bulk streamwise velocity $U_{b}$. The streamwise and spanwise dimensions of the domain are chosen to be $2 \pi \delta$ and $\pi \delta$, respectively. The bulk Reynolds number is $\operatorname{Re}=\delta U_{b} / \nu$, where $\nu$ is the kinematic viscosity. The boundary layer Reynolds number is defined as $\operatorname{Re}_{\tau}=u_{\tau} \delta / \nu$, where $u_{\tau}$ is the shear velocity.

The bulk Reynolds number was chosen to be $\operatorname{Re}=10935$, which corresponds to $\operatorname{Re}_{\tau}=590$ as in $M$ MM. All cases have been averaged over an advective time of at least $500 \delta / U_{b}$ at a time step of $\Delta t=0.02 \delta / U_{b}$. In addition, quantities were averaged over statistically homogeneous directions, $x$ and $z$, and about the plane of symmetry $y=0$. In the most resolved case, the results for the mean flow are close to those of MKM. 


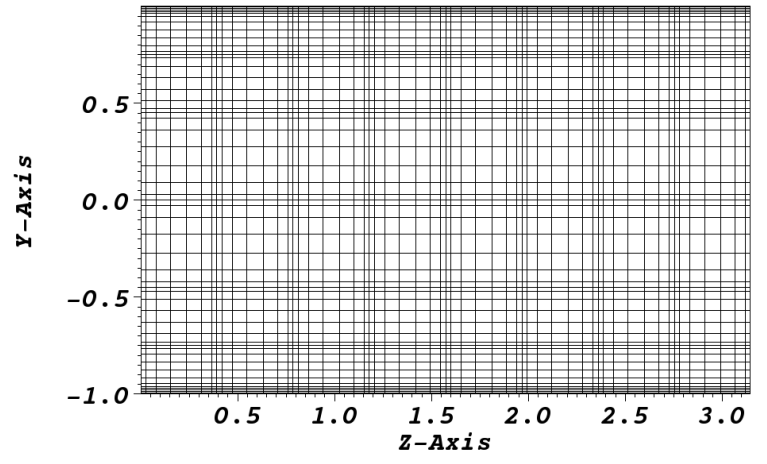

(a) M1: Cases A, B, H, I, J. $(8,8,8 ; 7)$

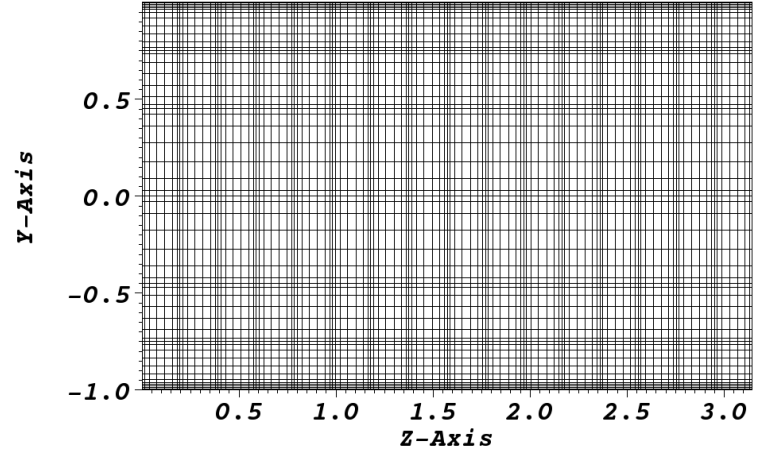

(b) M2: Case C. $(8,8,16 ; 7)$

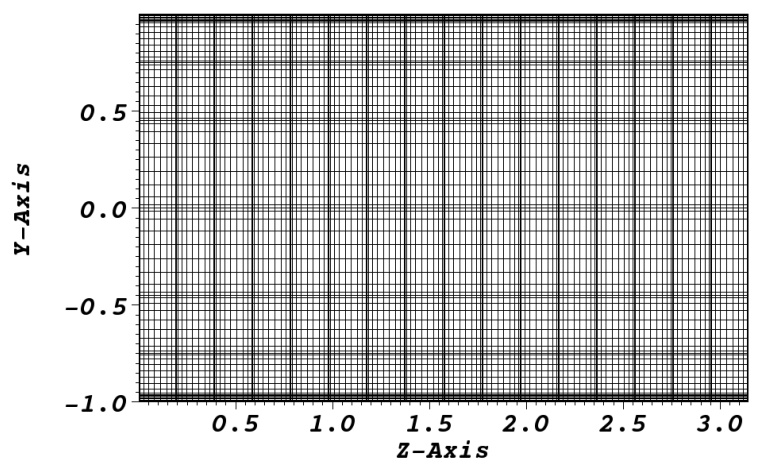

(c) M3: Cases D, E, F, G. $(8,8,16 ; 9)$

Figure 2: Meshes for channel flow with $\left(E_{x}, E_{y}, E_{z} ; N\right)$.

\subsection{Results}

In this section we consider how varying parameters of the computational method affects the solution: first, the Nek5000 filter parameters; second, spanwise resolution; third, streamwise resolution; and fourth, explicit SGS models. 
Table 1: Channel flow parameters for setups studied.

\begin{tabular}{|rrrrrrrclll|}
\hline \multicolumn{1}{|c|}{$\mathrm{Re}=10935, \mathrm{Re}_{\tau}=590$} \\
$E_{x}$ & $E_{y}$ & $E_{z}$ & $N$ & $\Delta x_{\max }^{+}$ & $y_{1}^{+}$ & $\Delta z_{\max }^{+}$ & $\tau_{w, m}\left(\times 10^{-3}\right)$ & $\Delta \tau_{\max }$ & $\alpha, M$ & Case \\
\hline 8 & 8 & 8 & 7 & 96.5 & 1.5 & 48.2 & 2.46 & 0.28 & $0.05,2$ & $\mathrm{~A}$ \\
8 & 8 & 8 & 7 & 96.5 & 1.5 & 48.2 & 2.89 & 0.49 & $0.005,1$ & $\mathrm{~B}$ \\
8 & 8 & 16 & 7 & 96.5 & 1.5 & 24.1 & 2.65 & 0.04 & $0.05,2$ & $\mathrm{C}$ \\
8 & 8 & 16 & 9 & 76.2 & 0.94 & 19.0 & 2.87 & 0.02 & $0.05,2$ & $\mathrm{D}$ \\
& & & & & & 2.88 & & & MKM \\
\hline
\end{tabular}

Each case computed used one of three meshes. The streamwise normal cross section of each mesh is shown in Fig. 2 and the cases that used each mesh are listed. Mesh M2 was created from M1 by doubling the number of spanwise elements. Mesh M3 has the same number of elements as M2 but with order $N=9$, instead of $N=7$. Shown are all of the spectral element nodes.

The base cases are summarized in Table 1, while additional cases are described in the text or summarized in Table 2. Listed is the number of elements in each direction $E_{x}, E_{y}, E_{z}$; the order of spectral elements $N$; the maximum streamwise mesh spacing $\Delta x_{\max }^{+}$; the first grid point away from the wall $y_{1}^{+}$; the maximum spanwise mesh spacing $\Delta z_{\max }^{+}$; the mean wall shear stress $\tau_{w, m} ; \Delta \tau_{\max }$ the maximum deviation of $\tau_{w}$ from the mean, defined below; the filter level $\alpha$; and number of modes filtered $M$. The values $(\cdot)^{+}$shown in the table are normalized by the mean wall shear stress $\tau_{w, m}$ of MKM, so that cases calculated on the same mesh have the same wall coordinates, despite having different values of mean wall shear stress. However, in the figures presented all data have been normalized by the values of the corresponding calculation.

\subsubsection{Effect of Filter}

To examine the effect filter strength, we compare two setups of the same resolution. The high-filter case A had the filter parameters $\alpha=0.050, M=2$. The low-fiter case B had $\alpha=0.005, M=1$. These are summarized in Table 1 .

In the spanwise wall shear stress profile (Fig. 3a), we clearly see the element boundaries at each spike. This effect is due to the lack of strong continuity requirements of the spectral element method at the element boundaries (Section 3). The wall shear stress $\tau_{w}$ is computed from the derivatives, which are not required to be continuous across element boundaries. We also see that the variation in the profile is of the same form but that the high filter shows a decrease in the magnitude of the spikes.

We quantify the effect of filtering on the wall shear stress $\tau_{w}$ by the maximum peak deviation from the mean in the spanwise variation of the wall shear stress $\Delta \tau_{\max }:=\max _{x \in \Omega}\left|\tau_{w}(x)-\tau_{w, m}\right| / \tau_{w, m}$. The value is $28 \%$ for the high filter, and the value is $49 \%$ for the low filter. These values may seem to indicate that 
a higher filter is better. However, by comparing the mean wall shear stress values with the MKM case, we see that this is not true. The high-filter case predicts $\tau_{w, m}=2.46 \times 10^{-3}$, and the low-filter case predicts $\tau_{w, m}=2.89 \times 10^{-3} ;$ according to MKM, $\tau_{w, m}=2.88 \times 10^{-3}$. While greater filtering of the solution decreases the spikes in the streamwise variation of $\tau_{w}$, it also inaccurately reduces the mean value.

The effects of a low value of $\tau_{w, m}$ are also noticed by looking at the mean streamwise velocity profile (Fig. $3 \mathrm{~b}$. Here we see that the underprediction of $\tau_{w, m}$ in the case of the high filter results in overpredicting the mean streamwise velocity. We also see that the low filter fits the expected law of wall quite well. However, using the mean wall shear stress as a measure of the fidelity of the result is not recommended because, as we see in the spanwise profile of $\tau_{w}$, an accurate mean does not indicate that the result is correct throughout the domain.

\subsubsection{Effect of Spanwise Resolution}

We compare the effects of spanwise resolution by considering cases A, C, and D, where the filtering is the same but the mesh parameters change. Case $\mathrm{C}$ has had the number of spanwise elements doubled, and case D has further had the polynomial order increased from $N=7$ to $N=9$. The increase in elements improved $\tau_{w, m}$ modestly, raising it from 2.46 to 2.65 . However, it reduced the spikes in $\tau_{w}$ from $\Delta \tau_{\max }=0.28$ to $\Delta \tau_{\max }=0.04$. The mean streamwise profile is improved marginally. The increase in element order from case $\mathrm{C}$ to case $\mathrm{D}$ further reduces $\Delta \tau_{\max }$, while predicting a $\tau_{w, m}$ very close to the DNS value. The mean streamwise profile of $\mathrm{D}$ is also very close to the expected value.

The turbulent kinetic energy $k^{+}=\frac{1}{2}\left(\overline{u^{2}}+\overline{v^{2}}+\overline{w^{2}}\right) / u_{\tau}^{2}$ is calculated for each setup and shown in Fig. $3 \mathrm{~d}$ alongside the MKM data. Another effect of underresolution can be observed in the overprediction of $k^{+}$near the wall. The effect is substantial in the underresolved cases A and C. In the more resolved case $\mathrm{D}, k^{+}$only slightly overpredicts the peak. In all cases, $k^{+}$is underpredicted away from the wall. Also, one can see in Fig. $3 \mathrm{~d}$ an indicator of the lack of resolution in the $y$ direction; the turbulent kinetic energy contains peaks in case $\mathrm{B}$ near element boundaries at $y^{+} \approx 140$ and $y^{+} \approx 320$, similar to what was seen with the wall shear stress.

While streamwise and wall-normal resolution has been increased from case C to case D, the spanwise variation in the mean value of the wall shear stress is most heavily dependent on the spanwise resolution. In the following section, the stress will be shown to vary little with the streamwise resolution. In fact, the resolution in the streamwise direction will affect the streamwise variation of the wall shear stress, but that is not primary focus of the present comparisons. 


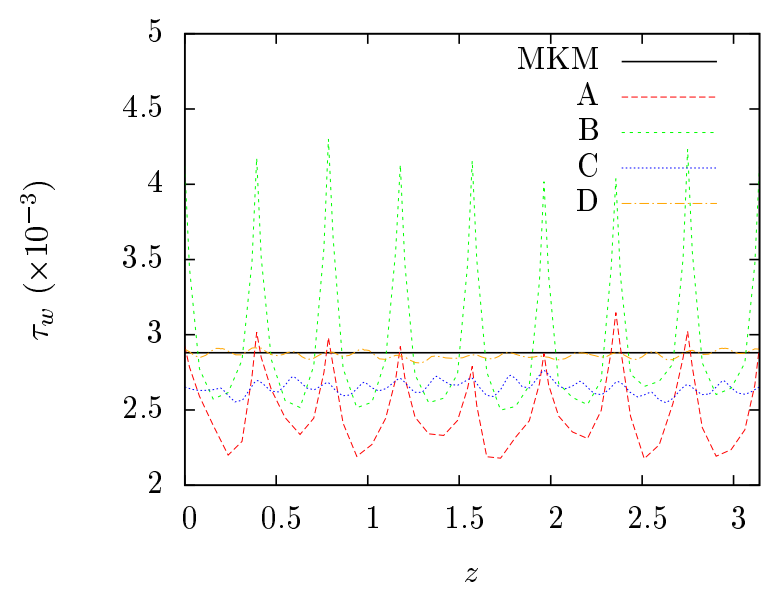

(a) Spanwise wall shear stress

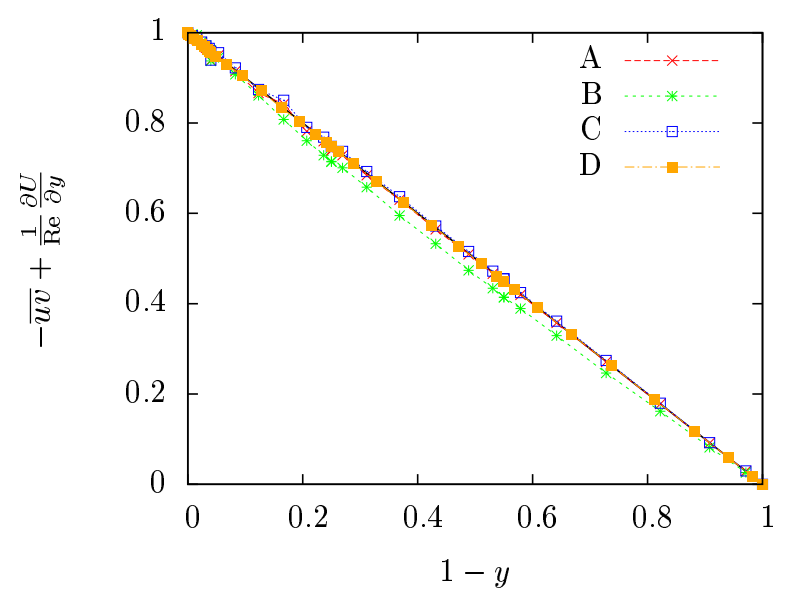

(c) Total shear stress

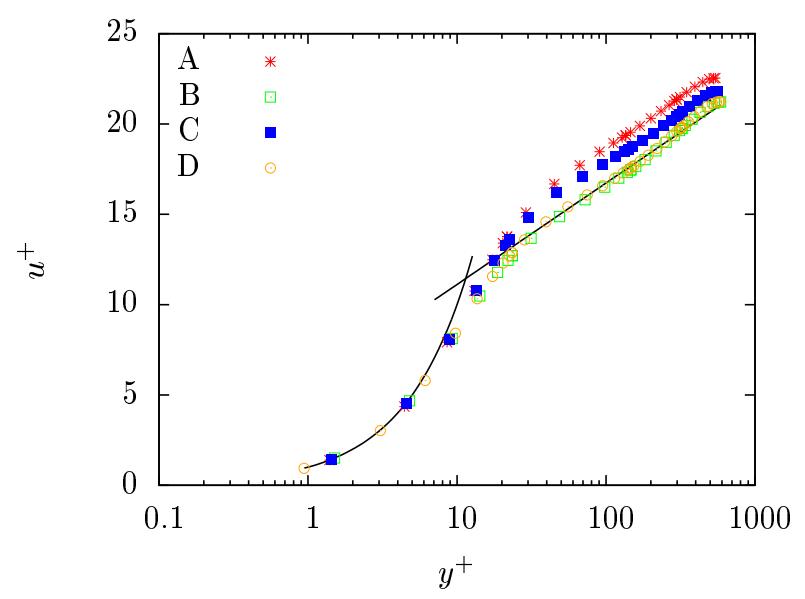

(b) Mean velocity profile

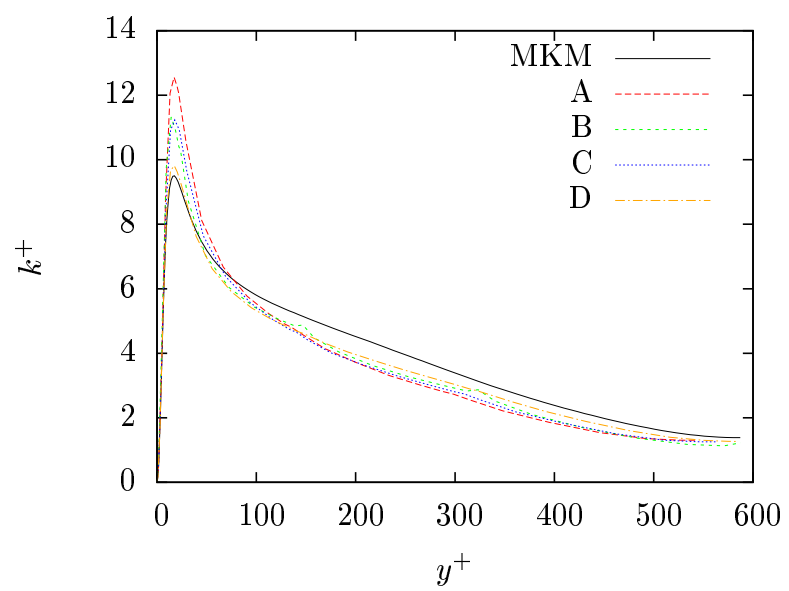

(d) Turbulent kinetic energy, $k^{+}$

Figure 3: High-filter and low-filter cases. 


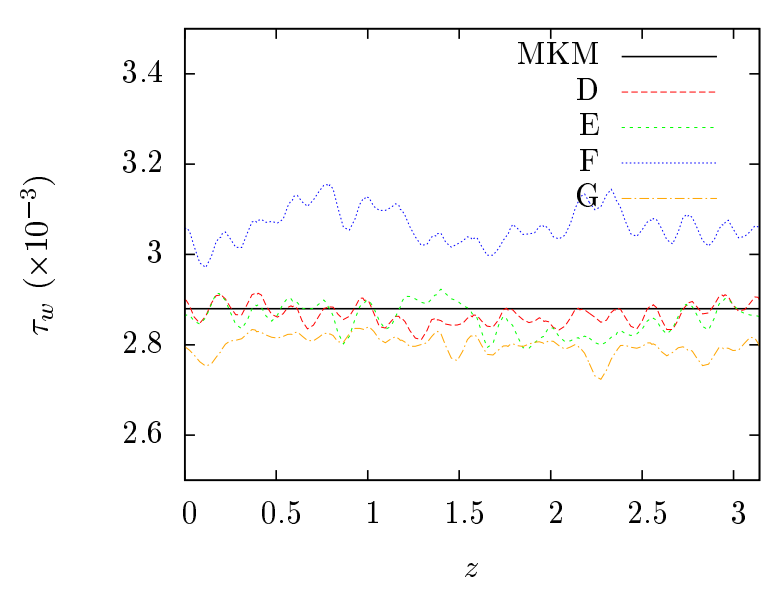

(a) Spanwise wall shear stress

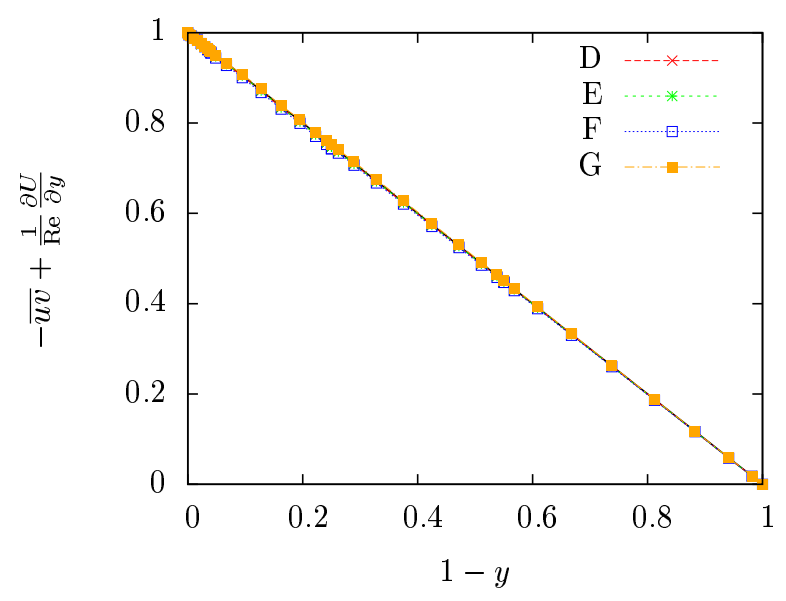

(c) Total shear stress

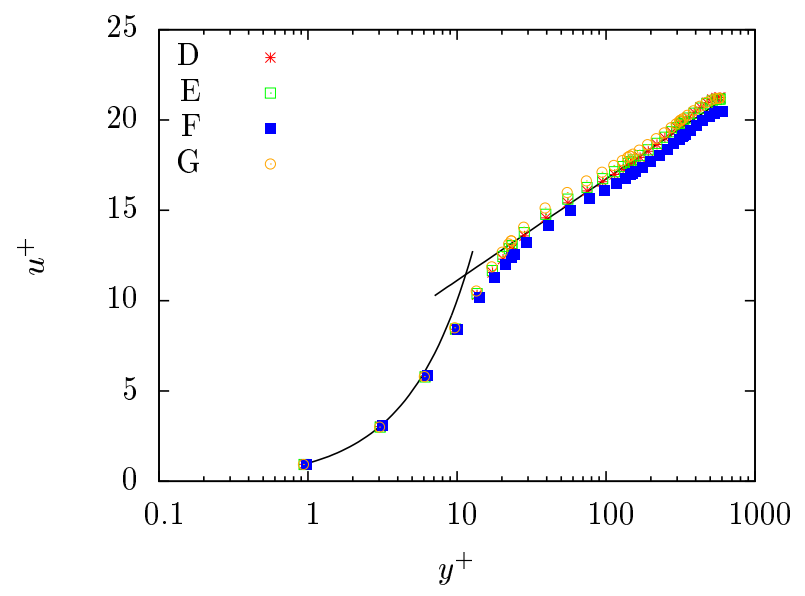

(b) Mean velocity profile

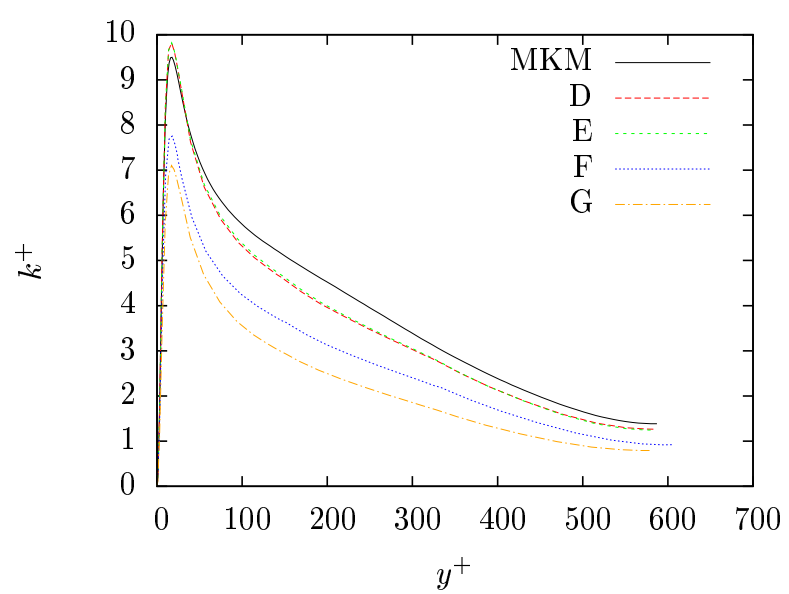

(d) Turbulent kinetic energy, $k^{+}$

Figure 4: Highest resolution mesh, stretched by a factor $\alpha_{z}$. Cases $\mathrm{E}$ and $\mathrm{F}$ have $\alpha_{z}=5 / 4$, and case $\mathrm{G}$ has $\alpha_{z}=3 / 2$. Additionally, case $\mathrm{F}$ has filtering turned off in wall elements. 


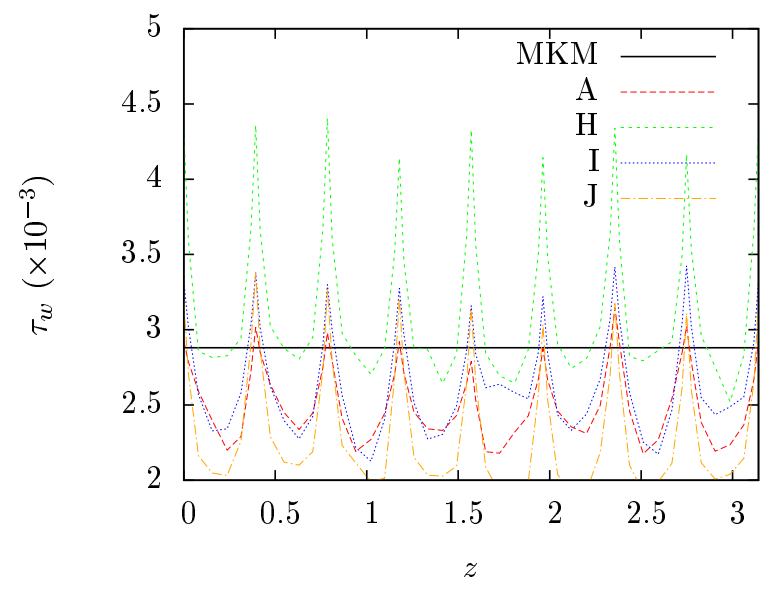

(a) Spanwise wall shear stress

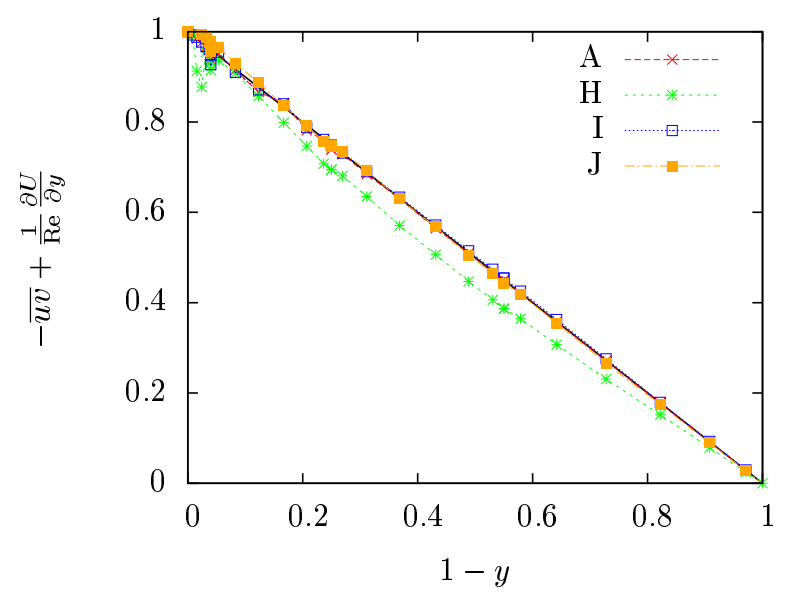

(c) Total shear stress

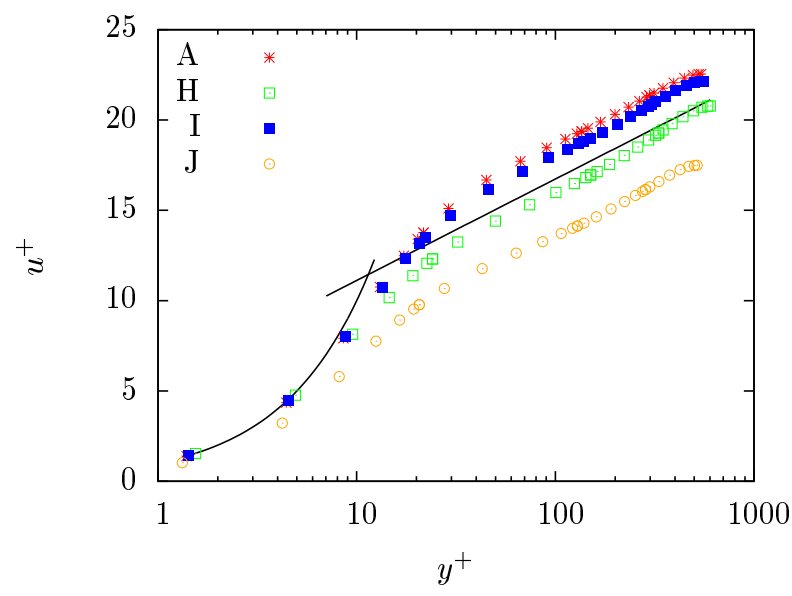

(b) Mean velocity profile

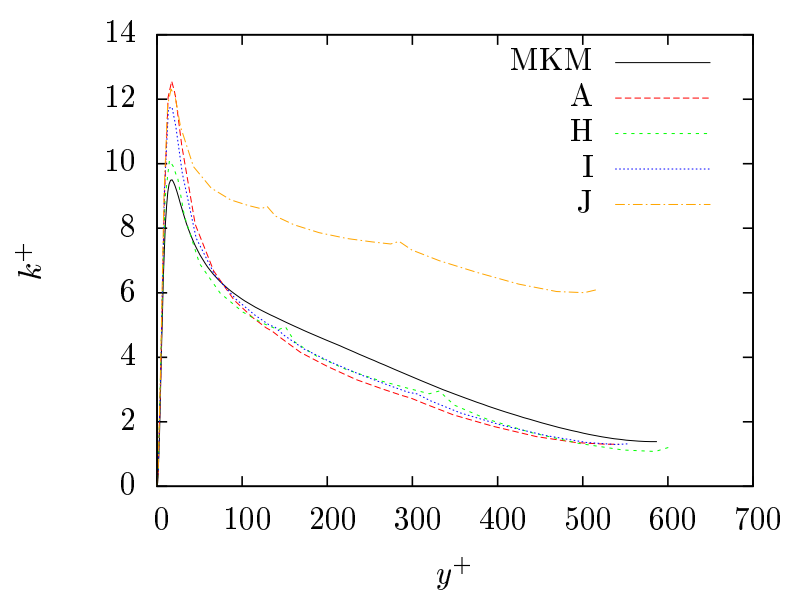

(d) Turbulent kinetic energy, $k^{+}$

Figure 5: Low-resolution mesh with various filters: A - standard Nek5000 filter with $\alpha=0.05, M=1 ; \mathrm{H}$ - dynamic Smagorinsky; I - same as A but with no filtering in the spanwise direction; J - hyperviscosity formulation.

\subsubsection{Streamwise Resolution}

In addition to comparing the effects of spanwise resolution, we investigated the effects of decreasing the streamwise resolution of case D. Using the same mesh, we extended the domain in the streamwise direction by a factor $\alpha_{z}$, reducing the streamwise resolution relative to case $\mathrm{D}$ by $1 / \alpha_{z}$. The results of three cases of this kind are summarized in Table 2 .

In cases $\mathrm{E}$ and $\mathrm{G}$ we see that the decrease in resolution leads to more filtering and, thus, a lower $\tau_{w, m}$. This was clear from considering the setups above. As a result, the mean profile is seen to lie above the expected law of the wall (Fig. 4b). We note that the effect on $\tau_{w, m}$ is modest, even for a decrease in 
streamwise resolution of $1 / 3$. In addition, $\Delta \tau_{\max }$ does not change dramatically from that for case $\mathrm{D}$. Cases $\mathrm{E}$ and $\mathrm{G}$ are still within the recommended level of resolution for an LES, indicating that within these bounds the spanwise variation of mean quantities does not vary strongly with streamwise resolution. In spite of this, the underprediction of kinetic energy in case $\mathrm{G}$ demonstrates the need for more resolution.

It is worth noting that we are considering the effects of streamwise resolution on spanwise variation, not streamwise variation. While not shown in this article, the streamwise profile of $\tau_{w}$ shows a behavior similar to the spanwise profile, exhibiting spikes at the element boundaries.

The effect of removing filtering along the boundary was also considered. This was achieved by manually turning off the filtering mechanism for all elements that touch the no-slip boundary. Case F represents this setup. We see that the $\tau_{w, m}$ is overpredicted. This overprediction leads to the mean streamwise velocity profile falling below the expected values (Fig. $4 \mathrm{~b}$. This result is likely due to the highest wavenumber, unstable modes gathering energy, since they are not removed by the filter.

\subsubsection{SGS Models}

To determine whether the mesh imprinting seen in the underresolved case was characteristic of the specific LES filter used, we simulated the channel flow with two other subgrid scale models and a modification of the standard Nek5000 spectral filter. The first, case H, used the dynamic Smagorinsky model. The second, case I, used an anistropic spectral filter that applied only in the streamwise and wall-normal directions. The third, case J, used a hyperviscosity formulation, where $\nabla^{2 \alpha}$ terms for both $\alpha=1$ and $\alpha=2$ are included. The weight of the $\alpha=2$ term is set independently of the viscosity.

We consider only the qualitative effects shown in Fig. 5. We notice that the results of the anisotropic filter in case I differ only slightly from case A. Case H produces results similar to the low-filter case B, where $\tau_{w, m}$ is slightly overpredicted and $\Delta \tau_{\max }$ is large. Case $\mathrm{J}$ is observed to be inaccurate.

The main observation is that the effect of underresolution is independent of the exact subgrid scale model used. The same mesh imprinting is seen in each case.

Table 2: Channel flow parameters for setups studied

\begin{tabular}{|c|c|c|c|c|}
\hline \multicolumn{5}{|c|}{$\operatorname{Re}=10935, \operatorname{Re}_{\tau}=590$} \\
\hline 1 & 76.2 & 2.87 & $\mathrm{Y}$ & $\mathrm{D}$ \\
\hline $5 / 4$ & 95.3 & 2.86 & $\mathrm{Y}$ & $\mathrm{E}$ \\
\hline $5 / 4$ & 95.3 & 3.06 & $\mathrm{~N}$ & $\mathrm{~F}$ \\
\hline $3 / 2$ & 114 & 2.80 & $\mathrm{Y}$ & $\mathrm{G}$ \\
\hline & & 2.88 & & MKM \\
\hline
\end{tabular}




\subsection{Discussion}

For wall-resolved LES, the recommended level of resolution is usually given as [10 23]

$$
50 \leq \Delta x^{+} \leq 150, \Delta y_{1}^{+}<1,15 \leq \Delta z^{+} \leq 40
$$

for "flat platelike" configurations where the $x$ direction is streamwise, the $y$ direction is wall normal, $y_{1}^{+}$is the first point away from the wall, and the $z$ direction is spanwise (homogeneous).

We have found that these recommendations were sufficient criteria to produce the correct mean streamwise velocity, total shear stress, and mean wall shear stress. However, they were not sufficient to correctly reproduce the wall shear stress across the entire spanwise direction. The cases with $\Delta z_{\max }^{+}=48.2$ showed clear mesh imprinting on the wall shear stress values due to the local filtering within each element. For the

cases with $\Delta z_{\max }^{+} \simeq 20$, we found that the spikes were greatly reduced. The most resolved case, which is well within the resolution guidelines, still showed minimal mesh imprinting in the wall shear stress. A more stringent requirement of $\Delta z_{\max }^{+} \leq 20$ is more appropriate for the resolution of a spanwise-varying quantity, such as $\tau_{w}$. However, a requirement such as maximum grid spacing does not take into account the increased wave number resolution inherent in high-order methods. As demonstrated in Section 5 , a higher order is preferable to a fine mesh.

We have also found that increased filter amplitudes are not effective in reducing the resolution requirements. In fact, while increasing the regularity of the solution, high-amplitude filters adversely affect the calculation by removing too much of the near-wall turbulent energy. The end result is less accurate mean flow values. However, with decreased filter amplitudes, the local error quantified in $\Delta \tau_{\max }$ increases, which is also not desirable. One cannot escape from the effects of underresolution.

\section{Rod Bundle Flow}

Turbulent flow through rod bundles is composed of complex energy production and transport mechanisms that arise from the interactions of the flow with the walls and with gaps, leading to flow patterns similar to

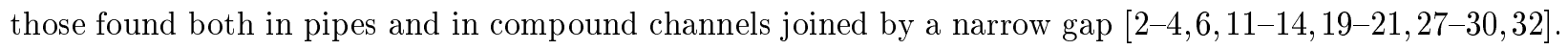
The problem has been studied with numerous experiments and computations by the nuclear engineering community because of its application to safe and efficient nuclear hydraulic design.

In 1998, Krauss and Meyer, hereafter KM, published results of a systematic study they conducted on an 
experimental setup of a 37 rod bundle [16]. Their bundle consisted of an array of triangular pitch that they could vary. They reported on two studies in this bundle. In the one study, the rods were arranged with a pitch-to-diameter ratio of $P / D=1.12$, wall-to-diameter ratio of $W / D=1.06$, and bulk Reynolds number $\operatorname{Re}_{\mathrm{b}}=64590$. In the other study the parameters were $P / D=1.06, W / D=1.03$, and $\operatorname{Re}_{\mathrm{b}}=38754$. We are concerned with the former. In their study, they measured mean flow properties for both momentum and heat in a central channel of the rod bundle, including turbulence intensity of the velocities, turbulent kinetic energy, wall shear stress, and Reynolds stresses. Measurements were made by hot wire anemometry using a three-wire probe. In further investigations, they provided substantial evidence for the existence of a gap vortex street responsible for strong interchannel mixing through the rod gaps.

\subsection{Computational Model}

In our study, we conducted calculations of the flow around a single rod in a periodic array. Five cases are compared. The domain and parameters were chosen to match those of a central rod in the bundle of the Krauss Meyer experiment and to contain two flow subchannels. The domain was periodic in the spanwise direction, so that opposite faces on the hexagon were connected. Streamwise periodicity was used. Geometry and coordinates are shown in Figure 6. Note that the geometry used in this study is equivalent to that used in other studies in which the narrow gap is located in the center of the computational domain between two subchannels. The pitch-to-diameter ratio of the array was $P / D=1.12$. The Reynolds number was $\operatorname{Re}=64590$ based on the bulk velocity $U_{b}=1$ and the hydraulic diameter of one flow channel, defined as $D_{h}:=\frac{6}{\pi}\left(\frac{P^{2}}{D^{2}}-1\right) D$. The length of the domain was $L=2 \pi D_{h}$ for cases A and B, and $L=8 \pi D_{h}$ for cases C, $\mathrm{D}$, and $\mathrm{E}$. The constant time step in advective time units $t_{a}=D / U_{b}$ was $\Delta t=0.0005 t_{a}$. Table 3 compares the units used in this study with those used by KM.

The initial condition for each case was a lower resolution solution interpolated to the higher resolution grid. The lower resolution solution was the result of many flow through times, over $200 t_{a}$.

Statistics were gathered over a time of 4-20 advective time units. Symmetries in the flow were used to further average the data of a minimal flow element. Therefore, the statistics were gathered over an effective time of 250-570 advective time units, depending on the particular case. For example, case D was run for $4.2 t_{a}$, such that after averaging over the homogeneous axial direction of length 9.63 and the twelve-fold symmetry in the spanwise plane, the solution was effectively averaged for a time of $485 t_{a}$.

In addition to those cases presented, three additional cases were simulated at the same grid resolution of case $\mathrm{D}$ but with different domain lengths. This was done to test the sensitivity of the solution to the domain 
length. Specifically the lengths were $L_{z}=4 \pi D_{h}, L_{z}=16 \pi D_{h}$, and $L_{z}=32 \pi D_{h}$. No difference was seen between the results for these additional simulations and those presented for case D.

The calculations were performed on an IBM Blue Gene/P with 32-bit PowerPC 450d cores at a clock speed $850 \mathrm{MHz}$. The highest resolution cases used 1024 nodes for a total of 4096 cores. Case D used 28800 total elements $\left(=40 /\right.$ minimal flow element $\times 12$ minimal flow elements $\left.\times E_{z}=60\right)$ and ran for 78 hours on 4096 cores for a total of 320,000 CPU hours.

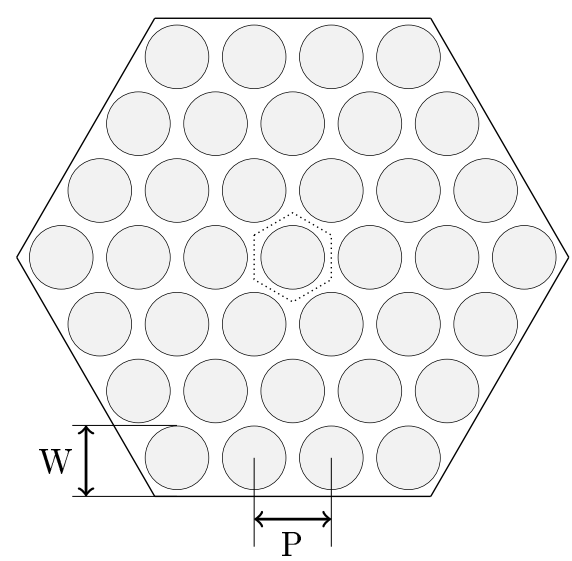

(a) Rod bundle configuration of Krauss and Meyer, with outline of single rod geometry indicated.

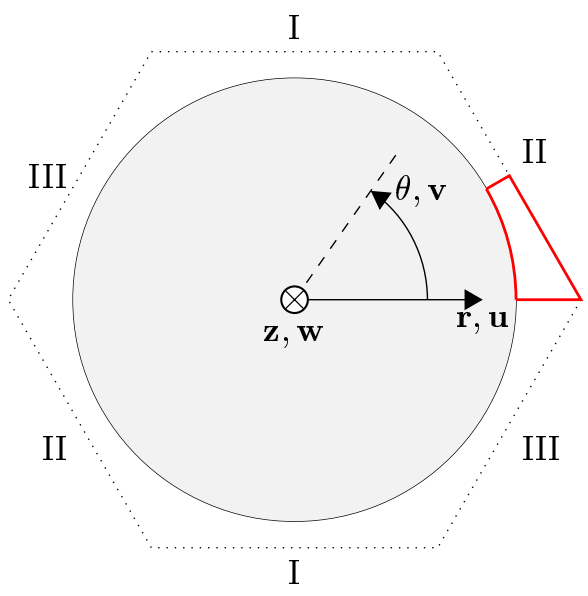

(b) Single rod in periodic array with periodicity in the plane labeled by I, II, III. Computational domain and coordinate system are shown and minimal flow element is indicated by the thick (red) line.

Figure 6: Cross section of domain.

Table 3: Dimensions

\begin{tabular}{|c|cc|}
\hline & Present & KM \\
\hline$D$ & 1 & $140 \mathrm{~mm}$ \\
$D_{h}$ & 0.38 & $53.6 \mathrm{~mm}$ \\
$U_{b}$ & 1 & $20.57 \mathrm{~m} / \mathrm{s}$ \\
$t_{a}$ & 1 & $6.8 \times 10^{-3} \mathrm{~s}$ \\
\hline
\end{tabular}

Table 4: Rod flow parameters for setups studied.

\begin{tabular}{|ccccccccc|}
\hline \multicolumn{8}{|c|}{$\mathrm{Re}=64590, P / D=1.12$, Filter: $\alpha=0.01, M=1$} \\
$E_{r}$ & $E_{\theta}$ & $E_{z}$ & $N$ & $r \Delta \theta_{\max }^{+}$ & $\Delta z_{\max }$ & $y_{1}^{+}$ & $\tau_{w, m}\left(\times 10^{-3}\right)$ & case \\
\hline 3 & 6 & 13 & 19 & 33 & 136 & 0.71 & 2.93 & $\mathrm{~A}$ \\
6 & 10 & 24 & 11 & 34 & 121 & 0.35 & 2.75 & $\mathrm{~B}$ \\
6 & 39 & 25 & 11 & 8 & 118 & 0.62 & 2.82 & $\mathrm{C}$ \\
3 & 15 & 15 & 19 & 13 & 119 & 0.62 & 2.83 & $\mathrm{D}$ \\
3 & 12 & 15 & 23 & 13 & 98 & 0.42 & 2.83 & $\mathrm{E}$ \\
& & & & & & 2.41 & $\mathrm{KM}$ \\
\hline
\end{tabular}




\subsection{Results}

We compare the solutions of five different calculations with the experimental data. The mesh configuration parameters for the calculations are summarized in Table 4. The number of radial elements is indicated by $E_{r}$, while $E_{\theta}$ indicates the number of azimuthal elements at the wall. A reduction in the number of azimuthal elements away from the wall is used in all cases except E (see Fig. 8a). The number of elements per $2 \pi D_{h}$ is given by $E_{z}$. The remaining values are the element order $N$; the maximum azimuthal spacing $r \Delta \theta_{\max }^{+}$; the maximum axial spacing $\Delta z_{\max }$; the distance of first point from the wall at $\theta=0$ in wall units; and the mean wall shear stress $\tau_{w, m}$. All cases are compared in Fig. 7 where $y$ is the radial distance from the wall of the rod, and $\hat{y}$ is the distance from the rod to the line of maximum velocity at $\theta=0$. In addition, further results of the flow calculations are presented for Case D. As before, $(\cdot)^{+}$indicates quantities that have been averaged and normalized by the local wall shear stress.

While in the following sections we continue to focus on the spanwise variation of the wall shear stress, it should be noted that the streamwise profiles of $\tau_{w}$ of all calculations presented are spiked at the element boundaries. In case a smooth streamwise wall shear stress distribution is desired, similar conclusions on a successful refinement strategy will apply.

\subsubsection{Effect of Polynomial Order}

In order to compare the effectiveness of using higher-order spectral elements versus lower-order spectral elements, two different cases with the same number of points in the spanwise direction are compared. The higher-order case A consists of a mesh of 3 elements radially and 6 elements spanwise in the minimal flow element, all of order $N=19$. The lower-order case B consists of a mesh of 6 elements radially and 10 elements spanwise in the minimal flow element, all of order $N=11$.

The mean streamwise velocity profile is found to be robust to changes in the resolution. In Fig. 7b we compare the mean in the radial direction at $\theta=0$ for all cases computed with the log law reported by KM, $u^{+}=2.5 \ln y^{+}+4.5$. Case B is slightly closer than A, despite the higher order using only 60 points radially compared with the lower-order case using 72 points radially. This result indicates that the solution with fewer points, A, is close to spatial convergence already. Also, we see that the higher-order elements can effectively capture the same solution with fewer grid points.

In Fig. $7 \mathrm{a}$, we see that the lower-order case has much greater spikes in $\tau_{w}$ over the the domain $\theta\left[^{\circ}\right]=[0,30]$. We note that while the higher-order case is not averaged for a sufficient amount of time to reach statistical convergence, the spikes have been shown to persist at the element boundaries regardless of the sample sizes 
in other cases. This result indicates that there are higher order modes of the solution that are better resolved through increasing element order (p-refinement) rather than number of elements (h-refinement).

Additionally, with the same filter parameters $\alpha=0.01, M=1$ the higher-order case is effectively filtering away less energy from the domain. A reason is that the filter is applied only at the last few modes, which are greater in the higher-order case (i.e., the critical wavenumber $k_{c}$ is higher with higher polynomial order).

\subsubsection{Refinement Strategy}

We now consider cases C, D, and E. Cases C and D used meshes that were refined by increasing the number of elements in the azimuthal direction near the wall. Away from the wall, the number of elements is smaller by a factor of 3 through the use of intermediate trapezoidal elements, shown in Fig. 8a. Case E does not employ this reduction away from the wall; the wall distribution of elements along the wall is maintained to the spanwise boundaries.

The main result of the refinement can be seen in Fig. 7a. Here we see that while $\mathrm{C}$ and D vary smoothly as $\theta, \mathrm{C}$ begins to depart from the experimental data near $\theta=10^{\circ}$. The profile of $\mathrm{C}$ also varies almost linearly with $\theta$, not as expected. It therefore overpredicts $\tau_{w}$ at $\theta=0$ and underpredicts in the region $\theta=\left[13^{\circ}, 20^{\circ}\right]$. Case $\mathrm{D}$ is closer but deviates from the data at $\theta=0, \theta=12.5$, and $\theta=30$. Case $\mathrm{E}$ is clearly the best. As noted in Table 4 the mean value of these three cases differs only slightly.

In all other measures shown, the three do not differ significantly. With regard to the total shear stress, they compare well to the expected value. The kinetic energy profile for all cases is similar.

\subsubsection{Additional Results}

Results for other flow properties are shown for case D.

The higher order moments at $\theta=0$ are shown in Fig. 9 . The wall-normal skewness behaves similarly to that of channel flow (KMM) near the wall, but far away from the wall it grows positively. The streamwise skewness varies significantly from channel flow, having two additional crossover points, near $y^{+} \approx 105$ and $y^{+} \approx 541$, where it takes on a small positive value. Above $y^{+}=541$, it maintains a negative value that varies with distance from the wall. One potential explanation for the region of positive skew would be the influence of ejection effects. However, these are seen in channel flow as well, without the positive skew. Another explanation is that within this geometry there exist secondary flows (Fig. 8b) that contribute to the bulk movement of slow speed of the fluid from the wall to the center of the subchannel. It is unclear whether either of these effects is methodological or physical, since the resolution away from the wall is relatively 
coarse. Further sampling does not affect the behavior.

The turbulent kinetic energy $k=\frac{1}{2}\left(u^{2}+v^{2}+w^{2}\right)$ is plotted in Fig. $8 \mathrm{c}$ in units of the mean flow speed $U_{b}$.

In Fig. 10, the distribution of instantaneous measurements of $(w, u)$ is plotted. The velocities have been normalized by the local shear velocity $u_{\tau}$. We see that the near-wall turbulence is essentially twodimensional, especially near the wide gap. There is also a dominance of production events in the wide gap region compared with the narrow gap, for example at $y^{+} \approx 52$ in the wide gap and $y^{+} \approx 49$ in the narrow gap. The distribution of $(w, v)$ becomes isotropic at smaller $y^{+}$in the narrow gap.

Figure 11 shows the energy spectrum calculated along the $z$ direction at the center of the narrow gap along with a line indicating the Kolmgorov $k^{-5 / 3}$ spectrum. The wavenumber $k=1$ corresponds to the length of the domain. The energy has been normalized by the total and the spectrum has been smoothed by binning. Results show that the inertial range is resolved over at least a decade. The spike at the highest wavenumber is a numerical artifact of the spacing of the points over which the FFT was performed, which was $L_{z} / 500$.

The components of the covariance tensor in the narrow gap are shown in Fig. 12, The velocity signal seems to decorrelate in a short time frame, unlike what is observed for tighter rod bundles [19].

Low-speed streaks can be seen in a plot of velocity contours on a cylindrical surface at $y^{+} \approx 2$ shown in Fig. 13. Streak spacing was found to be approximately $106 y^{+}$. Approaching $\theta=0$, the size of the streaks diminishes while the total number increases, indicating a significant increase in turbulence production. 


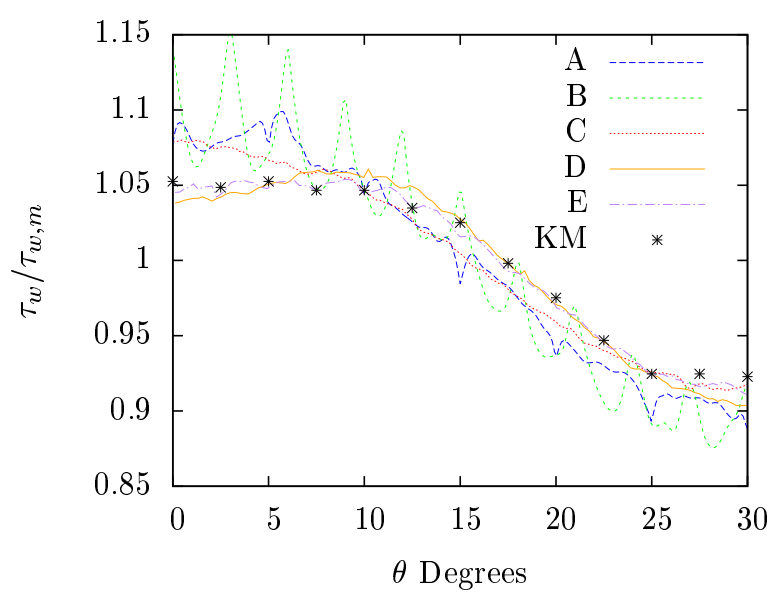

(a) Wall shear stress.

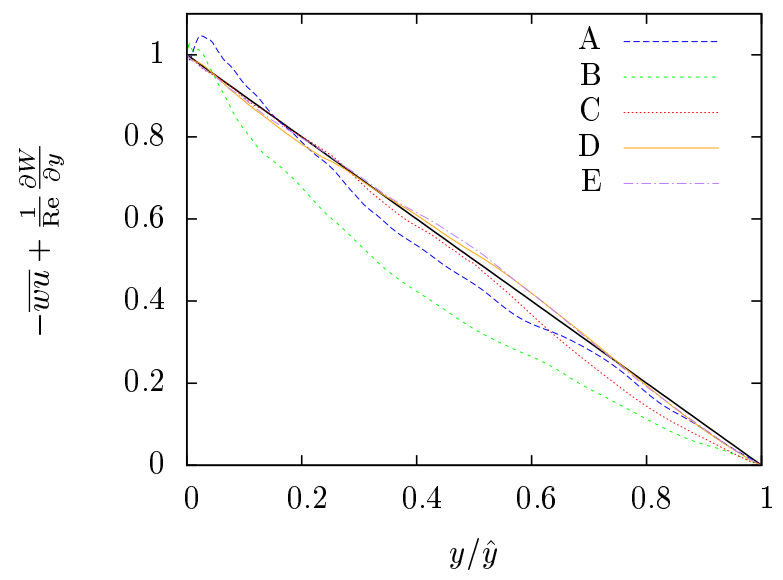

(c) Total shear stress.

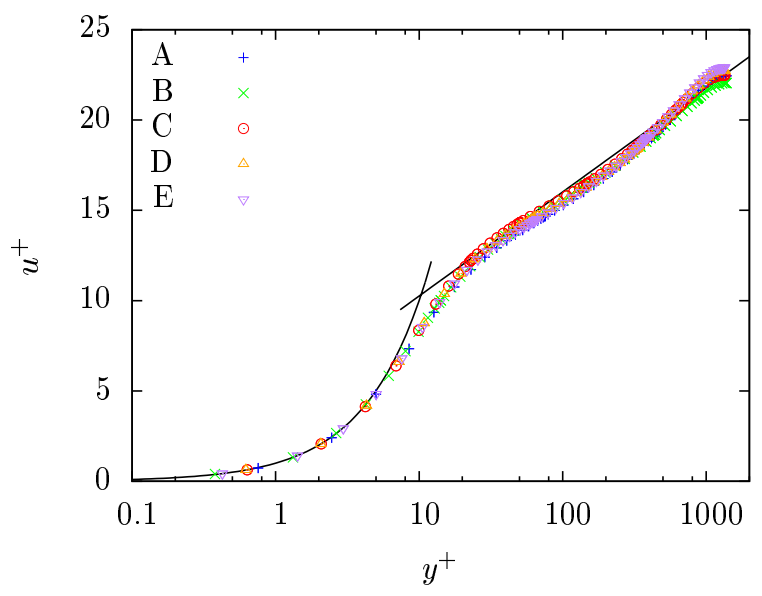

(b) Mean streamwise velocity.

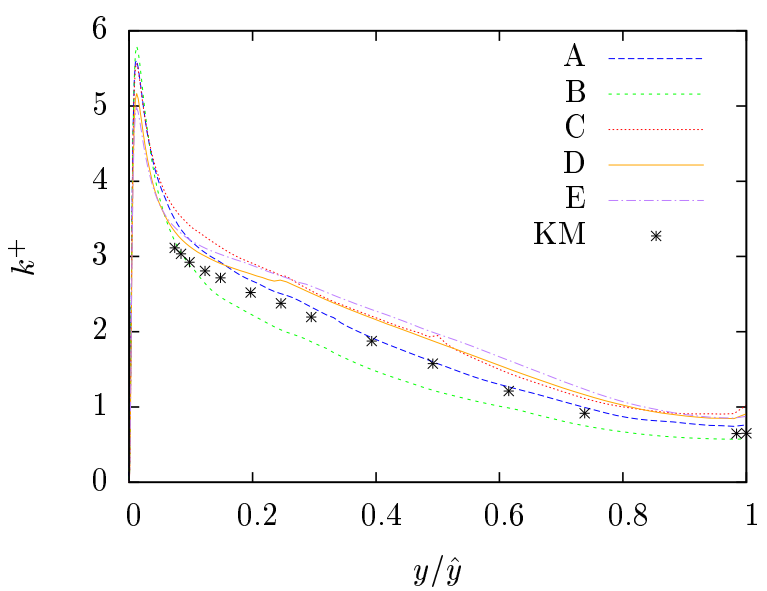

(d) Turbulent kinetic energy at $\theta=0$.

Figure 7: Comparison of mean flow quantities for rod. 

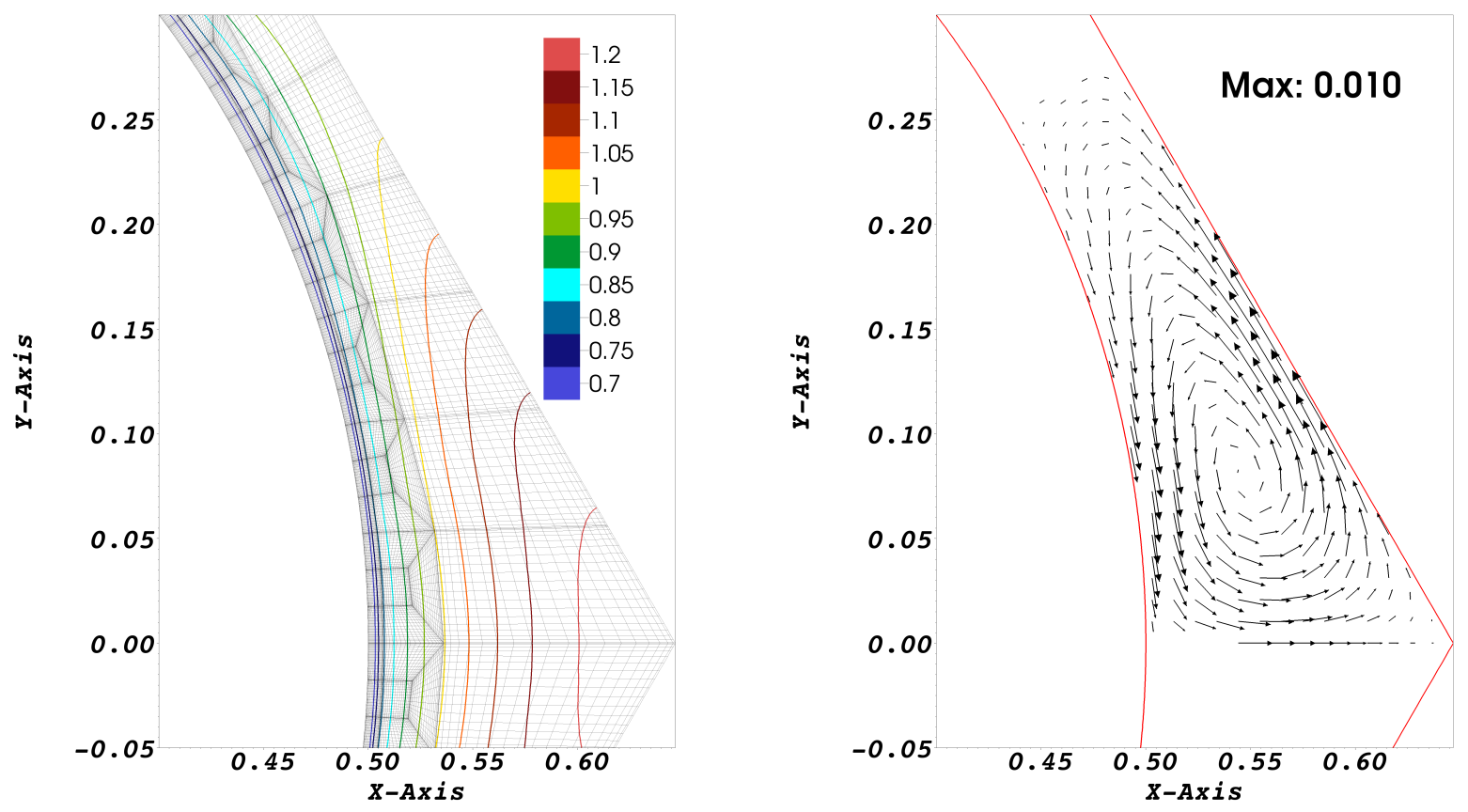

(a) Mean streamwise velocity $W$, normalized to bulk ve- (b) Secondary flow. Longest arrows indicate maximum velocity $U_{b}$. locity of $0.010 U_{b}$.

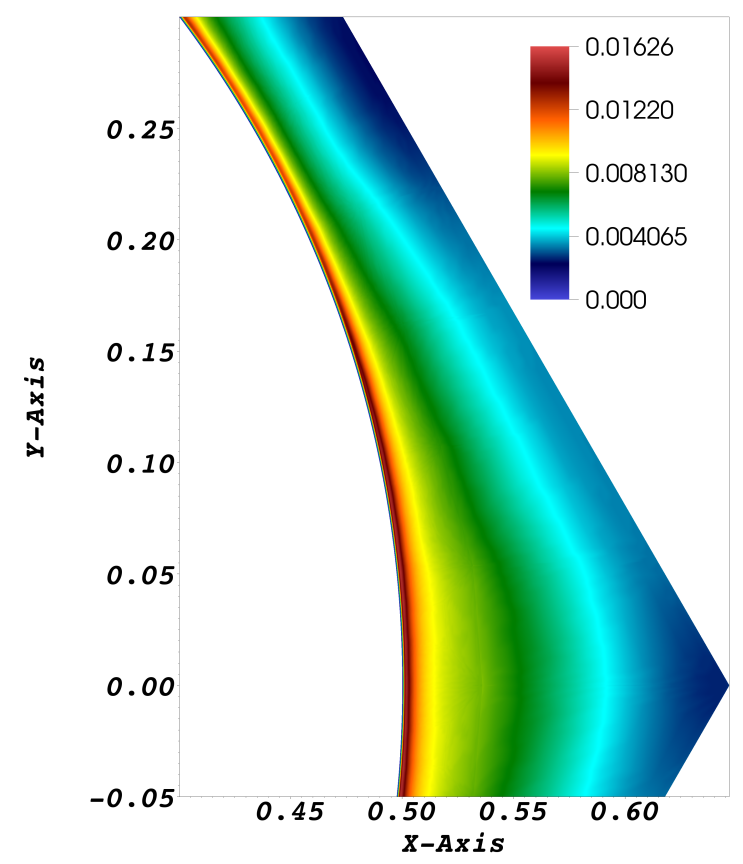

(c) Turbulent kinetic energy, normalized by bulk velocity $U_{b}$.

Figure 8: Mean flow properties. 


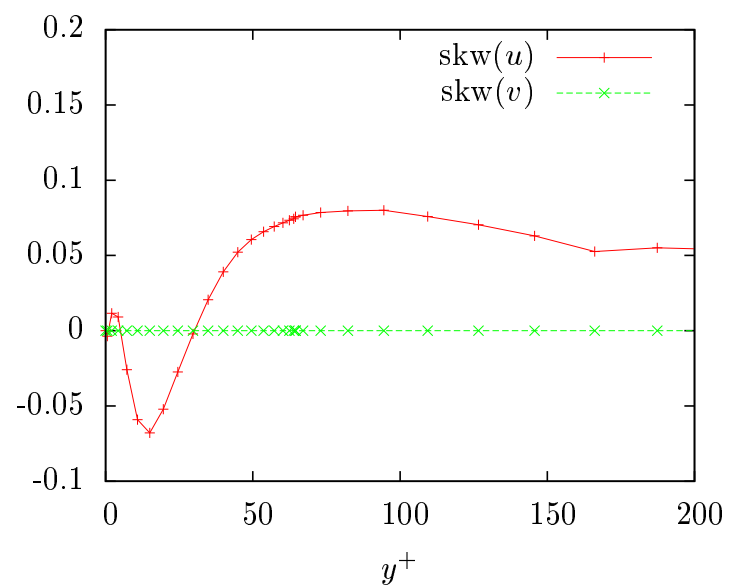

(a) Skewness for wall-normal and spanwise velocity components.

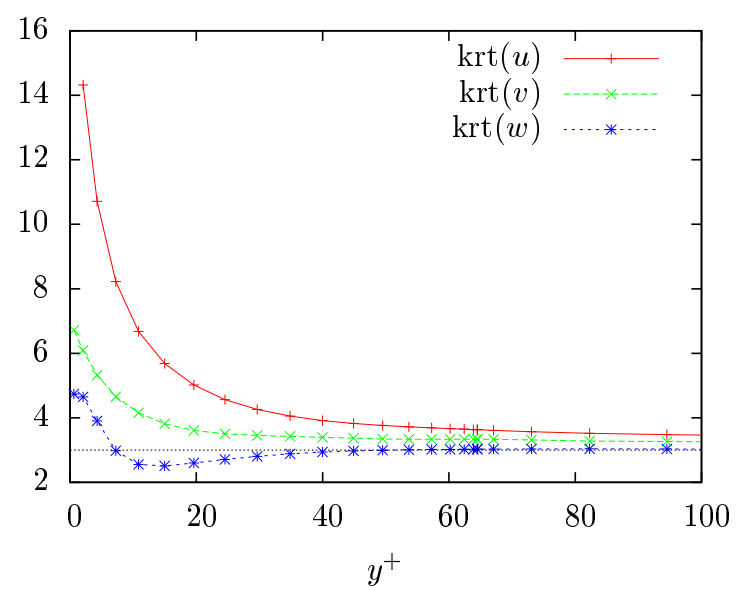

(c) Kurtosis for all three velocity components.

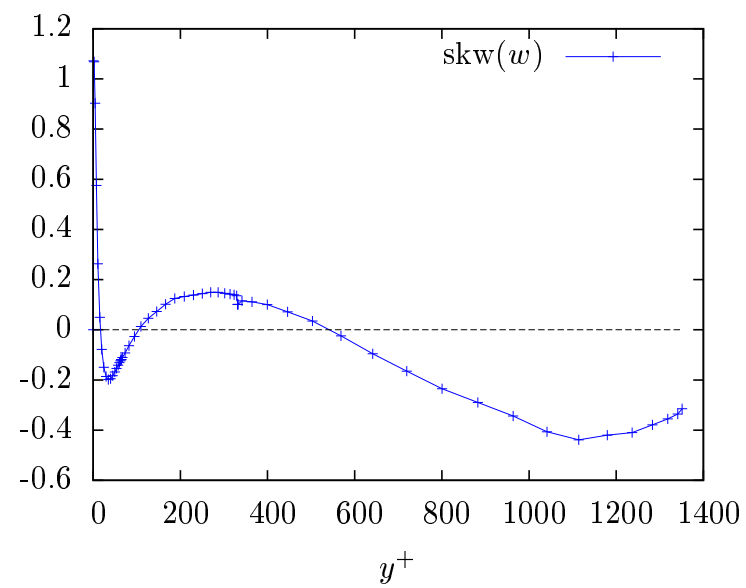

(b) Skewness for streamwise velocity component.

Figure 9: Higher-order moments of velocity at $\theta=0$. 


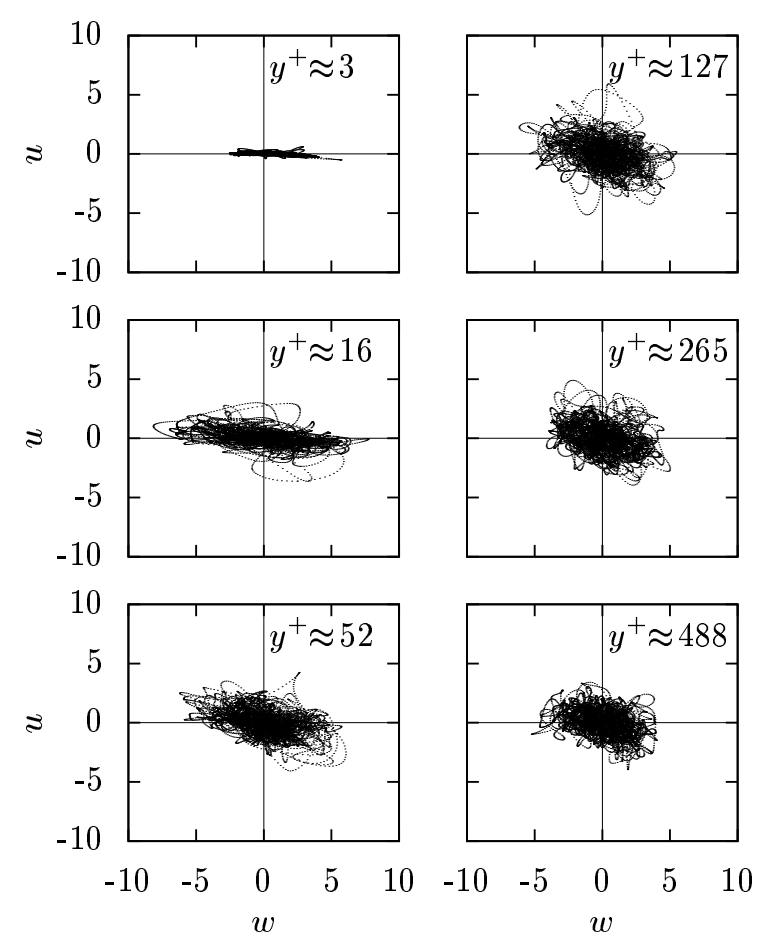

(a) Wide gap, $\theta=0^{\circ}$.
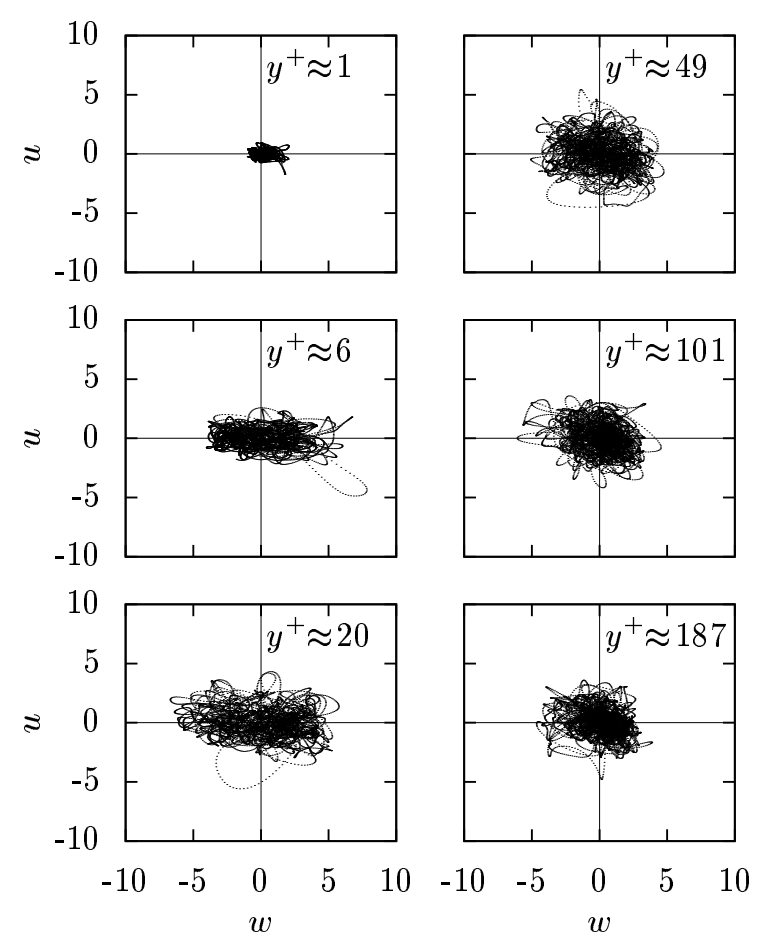

(b) Narrow gap, $\theta=30^{\circ}$.

Figure 10: Instantaneous $(w, u)$.

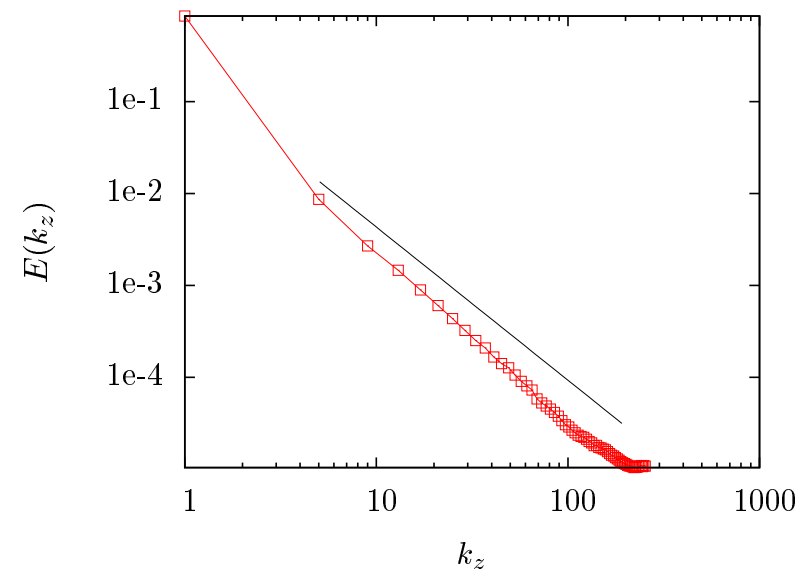

Figure 11: Streamwise energy spectrum in the narrow gap with $k^{-5 / 3}$ shown. 


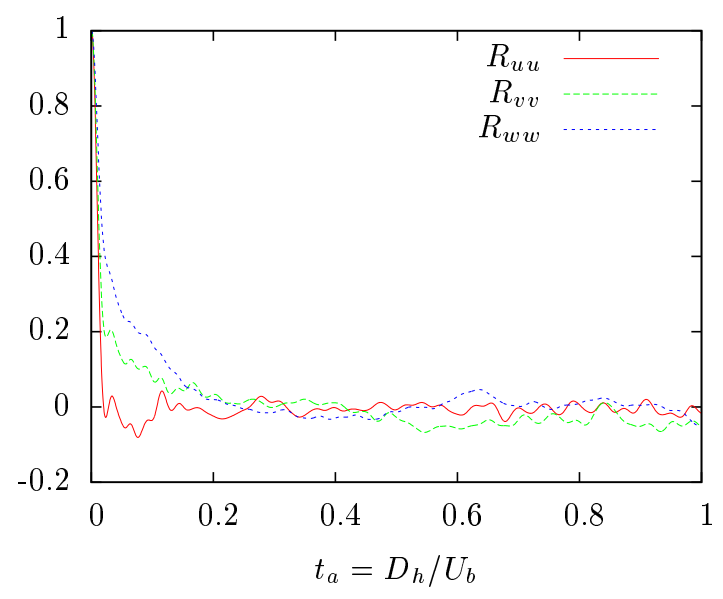

(a) Autocovariance

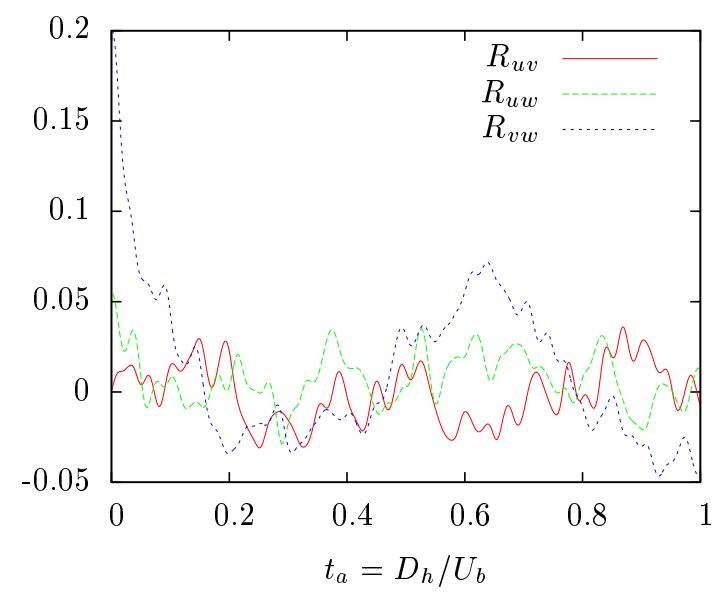

(b) Covariance

Figure 12: Time domain covariance of velocity fields at moniter point in the center of the narrow gap.

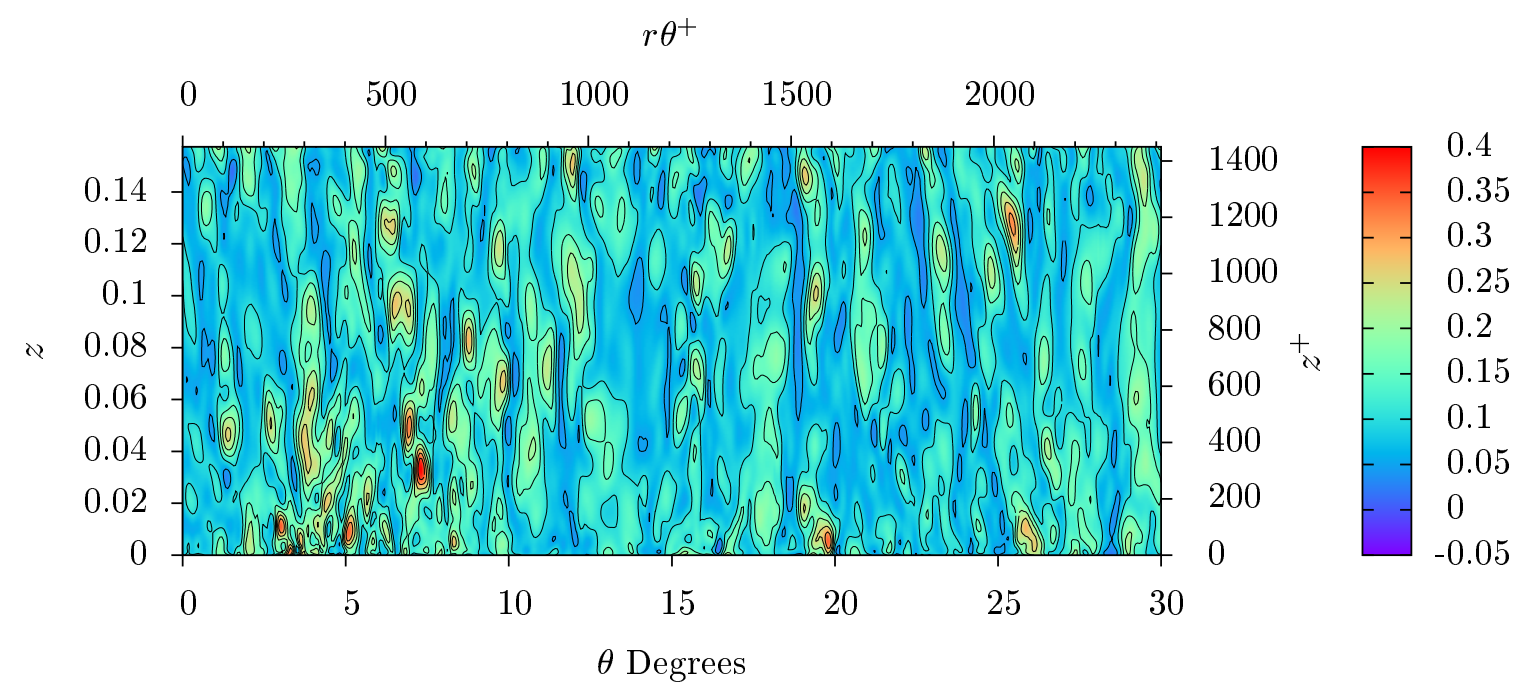

Figure 13: Streaks at $y^{+} \approx 2$.

\section{Conclusions}

A series of simulations for channel flow and an infinite-array rod bundle have been conducted with the spectral element code Nek5000. The effort was aimed at reproducing correctly the flow field while exploring the resolution requirements to solve accurately the wall shear stress.

We have found that the recommendations usually provided for channel flow (Eq. 11] [10 23] were sufficient criteria to produce the correct mean streamwise velocity, total shear stress, and mean wall shear stress. 
However, they were not sufficient to correctly reproduce the local wall shear stress in both rod bundles and channel flow.

In particular we found that the resolution in the direction where it is desired to resolve the local wall shear stress (e.g., spanwise in the present case) is crucial for producing accurate results. Such requirements lead to potentially much finer grids, if applied uniformly in the spanwise direction. A refinement strategy to maintain the resolution as coarse as possible in the bulk while meeting the resolution requirements in the near-wall region was devised (Fig. 8a).

The use of very high-order basis functions is found to be preferable to low order. For lower orders the resolution requirements tend to be higher. The most accurate results in the case of the rod were produced for 23rd-order polynomials using nearly half as many points near the wall as a comparable 11th-order case. Thus we find that generic guidelines of grid spacing are not the most useful for the problem considered here. We recommend that within the space of all possible grids that meet the general guidelines (Eq. 11), one should use as high a polynomial order basis function as necessary to resolve the regions of physical importance. In the case of high Reynolds number wall-bounded flows, this will be the region near the wall where strong shear produces the turbulence.

The simulations performed for a single rod calculation were in excellent agreement with available experimental results. The present guidelines and refinement approach are being used to perform a 37-rod simulation. Such simulation will be used to analyze in the detail the structure of turbulence in rod bundles and they will be the subject of a future article.

\section{Acknowledgments}

This work was supported in part by the Office of Advanced Scientific Computing Research, Office of Science, U.S. Dept. of Energy, under Contract DE-AC02-06CH11357.

Moreover, this research used resources of the Argonne Leadership Computing Facility at Argonne National Laboratory, which is supported by the Office of Science of the U.S. Department of Energy under contract DE-AC02-06CH11357.

\section{References}

[1] E. Baglietto and H. Ninokata. A turbulence model study for simulating flow inside tight lattice rod bundles. Nuclear Engineering and Design, 235(7):773-784, 2005. 
[2] F. Barato, S. Bailey, and S. Tavoularis. Measurements of frequencies and spatial correlations of coherent structures in rod bundle flows. Nuclear Engineering and Design, 236:1830-1837, 2006.

[3] M. Biemuller, L. Meyer, and K. Rehme. Large eddy simulation and measurement of the structure of turbulence in two rectangular channels connected by a gap. In Proc. 3rd Internat. Symp., Eng. Turbulence Modelling and Experiments. Elsevier, 1996.

[4] D. Chang and S. Tavoularis. Numerical simullation of turbulent flow in a 37-rod bundle. Nuclear Engineering and Design, 237:575-590, 2007.

[5] M. Deville, P. Fischer, and E. Mund. High-Order Methods for Incompressible Fluid Flow. Cambridge Monographs on Applied and Computational Mathematics. Cambridge University Press, 2002.

[6] W. Eifler and R. Nijsing. Experimental investigation of velocity distribution and flow resistance in a triangular array of parallel rods. Nuclear Engineering and Design, 5:22-42, 1967.

[7] P. Fischer. An overlapping Schwarz method for spectral element solution of the incompressible NavierStokes equations. Journal of Computational Physics, 1997.

[8] P. Fischer and J. Mullen. Filter-based stabilization of spectral element methods. Comptes rendus de l'Academie des sciences, Serie I Analyse numerique, 332:265-270, 2001.

[9] P. F. Fischer, J. W. Lottes, and S. G. Kerkemeier. Nek5000 Web page, 2008. http://nek5000.mcs.anl.gov.

[10] N. J. Georgiadis, D. P. Rizzetta, and C. Fureby. Large-eddy simulation: Current capabilities, recommended practices, and future research. Technical Memorandum NASA/TM-2009-215616, NASA, NASA Center for Aerospace Information, 7115 Standard Drive, Hanover, MD 21076-1320, July 2009.

[11] A. Gosset and S. Tavoularis. Laminar flow instability in a rectangular channel with a cylindrical core. Physics of Fluids, 18(4):044108, 2006.

[12] M. Guellouz and S. Tavoularis. Heat transfer in rod bundle subchannels with varying rod-wall proximity. Nuclear Engineering and Design, 132:351-366, 1992.

[13] J. Hooper. Developed single phase turbulent flow through a square-pitch rod cluster. Nuclear Engineering and Design, 60:365-379, 1980.

[14] J. Hooper and K. Rehme. Large-scale structural effects in developed turbulent flow through closelyspaced rod arrays. Journal of Fluid Mechanics, 145:305-337, 1984. 
[15] J. Kim, P. Moin, and R. Moser. Turbulence statistics in fully developed channel flow at low Reynolds number. Journal of Fluid Mechanics, 177:133-166, 1987.

[16] T. Krauss and L. Meyer. Experimental investigation of turbulent transport of momentum and energy in a heated rod bundle. Nuclear Engineering and Design, 180:185-206, 1998.

[17] Y. Maday, A. Patera, and E. Ronquist. An operator-integration-factor splitting method for timedependent problems: Application to incompressible fluid flow. Journal of Scientific Computing, 5:263$292,1990$.

[18] E. Merzari and H. Ninokata. Proper orthogonal decomposition of the flow in a tight lattice rodbundle. Nuclear Engineering and Design, 241(11):4621-4632, 2011. 13th International Topical Meeting on Nuclear Reactor Thermal Hydraulics.

[19] E. Merzari, H. Ninokata, and E. Baglietto. Numerical simulation of flows in tight-lattice fuel bundles. Nuclear Engineering and Design, 238:1703-1719, 2008.

[20] S. Möller. On phenomena of turbulent flow through rod bundles. Experimental Thermal an Fluid Science, 4(1):25-35, 1991.

[21] S. Möller. Single-phase turbulent mixing in rod bundles. Experimental Thermal an Fluid Science, $5(1): 26-33,1992$.

[22] R. D. Moser, J. Kim, and N. N. Mansour. Direct numerical simulation of turbulent channel flow up to $\operatorname{Re}_{\tau}=$ 590. Physics of Fluids, 11(4):943-945, April 1999.

[23] U. Piomelli and E. Balaras. Wall-layer models for large-eddy simulation. Annual Reviews of Fluid Mechanics, 34:349-374, January 2002.

[24] S. B. Pope. Turbulent flows. Cambridge university press, 2000.

[25] A. Quarteroni and A. Valli. Numerical approximation of partial differential equations. Springer, 2008.

[26] K. Rehme. Convective Heat Transfer over Rod Bundles, chapter 7. John Wiley \& Sons, 1987.

[27] K. Rehme. The structure of turbulent flow through rod bundles. Nuclear Engineering and Design, 99:141-154, 1987.

[28] W. J. Seale. Turbulent diffusion of heat between connected flow passages. Nuclear Engineering and Design, 54:183-195, 1979. 
[29] W. J. Seale. Turbulent diffusion of heat between connected flow passages. Nuclear Engineering and Design, 54:197-209, 1979.

[30] W. J. Seale. Measurements and predicitions of fully developed turbulent flow in a simulated rod bundle. Journal of Fluid Mechanics, 123:399-423, 1982.

[31] C. team. The Cesar Codesign Center: Early results. In DOE Exascale Conference, 2012. https://cesar.mcs.anl.gov/sites/cesar.mcs.anl.gov/files/Technical

[32] A. C. Trupp and R. S. Azad. The structure of turbulent flow in triangular array rod bundles. Nuclear Engineering and Design, 32(1):47-84, 1975.

The submitted manuscript has been created by the University of Chicago as Operator of Argonne National Laboratory ("Argonne") under Contract DE-AC02-06CH11357 with the U.S. Department of Energy. The U.S. Government retains for itself, and others acting on its behalf, a paid-up, nonexclusive, irrevocable worldwide license in said article to reproduce, prepare derivative works, distribute copies to the public, and perform publicly and display publicly, by or on behalf of the Government. 